\section{Banking, insurance}

\section{Other banks balance sheet}

(Table 23.3)

The table includes the business of all monthly and quarterly reporting banks in the UK.

The Channel Islands and Isle of Man are not treated as part of the UK for statistical purposes. Banking institutions in the Channel Islands and Isle of Man no longer have the option of being within the UK banking sector and their business, along with the business of offshore island branches of UK mainland banks, is excluded from the figures within this table. Additionally, the business of the UK banking sector with offshore island residents and entities are classified as 'nonresidents'.

The table also contains details of business with building societies.

The aggregate balance sheet of the banking sector is reported on an accrual basis (accrued amounts that are payable and receivable are shown under liabilities and assets respectively). Additionally, acceptances are shown under both liabilities and assets.

The balance sheet of the Banking Department of the Bank of England is excluded from this table, and other banks business with the Issue Department is classified as 'UK banks'.

Data for 1999 reflect the acquisition of Birmingham Midshires Building Society by Halifax during that year.

Data for the end of 2000 reflect the entry of Bradford and Bingley plc to the banking sector during the year. Data for the end of 2000 also reflect the new reporting during the year of agency business as a result of collateral management via repurchase agreements (repos) and reverse repos.

\section{Bank lending to, and bank deposits from, UK residents}

\author{
(Tables 23.4 and 23.5)
}

These are series statistics based on the Standard Industrial Classification (SIC) 1992 (which was revised slightly in 2003).

Table 23.4. Until the third quarter of 2007, the analysis of lending covered loans, advances (including under reverse repos), finance leasing, acceptances and facilities (all in Sterling and other currencies) provided by reporting banks to their UK resident non-bank non-building society customers, as well as bank holdings of sterling and euro commercial paper issued by these resident customers. Following a review of statistical data collected, acceptances and holdings of sterling and euro commercial paper are no longer collected at the industry level detail with effect from fourth quarter 2007 data. Total lending therefore reflects loans and advances (including under reverse repos) only, from fourth quarter 2007 data.

Table 23.5 includes borrowing under sale and repo. Adjustments for transit items are not included.

Figures for both tables are supplied by monthly reporting banks and grossed to cover quarterly reporters. Following the transition of building societies' statistical reporting from the Financial Services Authority to the Bank of England on 1st January 2008, both tables will include data reported by building societies from the first quarter of 2008 onwards. They exclude lending to building societies and to residents of the Channel Islands and Isle of Man.

\section{Building societies}

(Table 23.13)

Building society figures are sourced from societies' annual returns and for each year relate to accounting years ending on dates between 1 February and 31 January of the following year. Figures are society-only as opposed to group consolidated.

\section{Consumer credit}

(Table 23.14)

Figures for net lending refer to changes in amounts outstanding adjusted to remove distortions caused by

revaluations of debt outstanding, such as write-offs. Class 3 loans are advanced under the terms of the Building Societies Act 1986 
A high proportion of credit advanced in certain types of agreement, notably on credit cards, is repaid within a month. This reflects use of such agreements as a method of payment rather than a way of obtaining credit. As from December 2006 the Bank of England has ceased to update the separate data on consumer credit provided by other specialist lenders, retailers and insurance companies previously contained in these tables. These categories have been merged into 'other consumer credit lenders'. 


\section{Banking, insurance}

\section{Bank of England Balance Sheet \\ - Liabilities and assets outstanding at end of period}

$£$ million

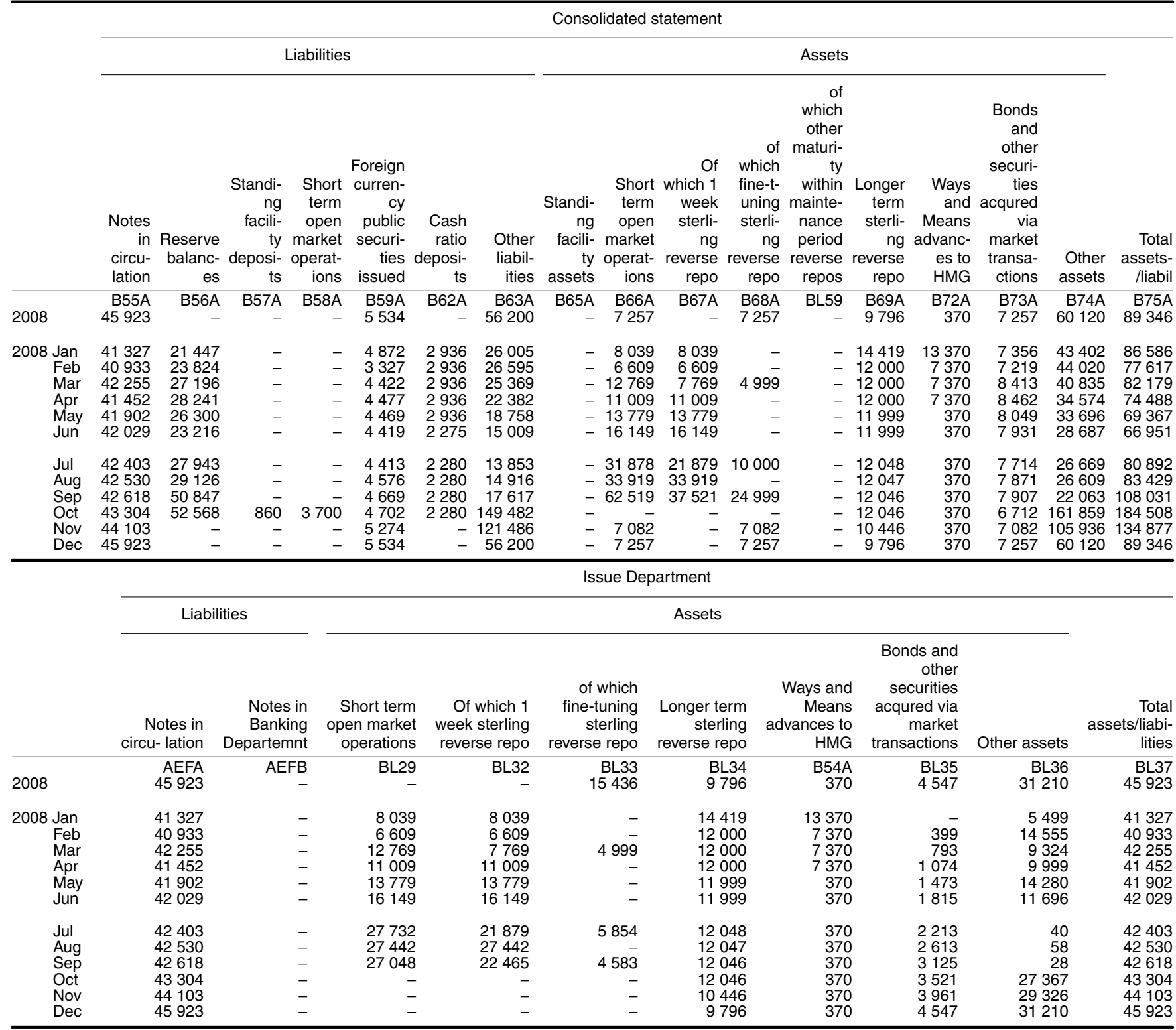

Banking Department

\begin{tabular}{|c|c|c|c|c|c|c|c|c|c|c|c|c|c|c|c|}
\hline & $\begin{array}{l}\text { Reserve } \\
\text { balances }\end{array}$ & $\begin{array}{r}\text { Standing } \\
\text { facility } \\
\text { deposits }\end{array}$ & $\begin{array}{r}\text { Short } \\
\text { term open } \\
\text { market } \\
\text { operatio- } \\
\text { ns }\end{array}$ & $\begin{array}{r}\text { Foreign } \\
\text { currency } \\
\text { public } \\
\text { securiti- } \\
\text { es issued }\end{array}$ & $\begin{array}{r}\text { Cash } \\
\text { ratio } \\
\text { deposits }\end{array}$ & $\begin{array}{r}\text { Other } \\
\text { liabilit- } \\
\text { ies }\end{array}$ & $\begin{array}{r}\text { Standing } \\
\text { facility } \\
\text { assets }\end{array}$ & $\begin{array}{r}\text { Short } \\
\text { term open } \\
\text { market } \\
\text { operatio- } \\
\text { ns }\end{array}$ & $\begin{array}{r}\text { Of which } \\
1 \text { week } \\
\text { sterling } \\
\text { reverse } \\
\text { repo }\end{array}$ & $\begin{array}{r}\text { of which } \\
\text { fine-tun- } \\
\text { ing } \\
\text { sterling } \\
\text { reverse } \\
\text { repo }\end{array}$ & $\begin{array}{r}\text { Longer } \\
\text { term } \\
\text { sterling } \\
\text { reverse } \\
\text { repo }\end{array}$ & $\begin{array}{r}\text { Bonds and } \\
\text { other } \\
\text { securiti- } \\
\text { es } \\
\text { acqured } \\
\text { via } \\
\text { market } \\
\text { transact- } \\
\text { ions }\end{array}$ & $\begin{array}{r}\text { Bank of } \\
\text { England } \\
\text { notes }\end{array}$ & $\begin{array}{l}\text { Other } \\
\text { assets }\end{array}$ & $\begin{array}{r}\text { Total } \\
\text { assets/l- } \\
\text { iabilitie }\end{array}$ \\
\hline 2008 & $\begin{array}{r}\text { BL38 } \\
-\end{array}$ & $\begin{array}{r}\text { BL39 } \\
-\end{array}$ & $\begin{array}{r}\mathrm{BL} 42 \\
-\end{array}$ & $\begin{array}{r}\text { BL43 } \\
5534\end{array}$ & $\begin{array}{r}\text { BL44 } \\
-\end{array}$ & $\begin{array}{r}\text { BL45 } \\
87410\end{array}$ & $\begin{array}{r}\text { BL47 } \\
-\end{array}$ & $\begin{array}{l}\text { BL48 } \\
7257\end{array}$ & $\begin{array}{r}\text { BL49 } \\
-\end{array}$ & $\begin{array}{l}\text { BL52 } \\
7257\end{array}$ & $\begin{array}{r}\text { B3J2 } \\
143273\end{array}$ & $\begin{array}{l}\text { BL53 } \\
7257\end{array}$ & $\begin{array}{r}\text { BL54 } \\
-\end{array}$ & $\begin{array}{r}\text { BL55 } \\
60120\end{array}$ & $\begin{array}{r}\text { BL56 } \\
74634\end{array}$ \\
\hline $\begin{array}{r}2008 \text { Jan } \\
\text { Feb } \\
\text { Mar } \\
\text { Apr } \\
\text { May } \\
\text { Jun }\end{array}$ & $\begin{array}{l}21447 \\
23824 \\
27196 \\
28241 \\
26300 \\
23216\end{array}$ & $\begin{array}{l}- \\
- \\
- \\
- \\
- \\
-\end{array}$ & $\begin{array}{l}- \\
- \\
- \\
- \\
- \\
-\end{array}$ & $\begin{array}{l}4872 \\
3327 \\
4422 \\
4477 \\
4469 \\
4419\end{array}$ & $\begin{array}{l}2936 \\
2936 \\
2936 \\
2936 \\
2936 \\
2275\end{array}$ & $\begin{array}{ll}31 & 504 \\
41 & 151 \\
34 & 694 \\
32 & 381 \\
33 & 039 \\
26705\end{array}$ & $\begin{array}{l}- \\
- \\
- \\
- \\
-\end{array}$ & $\begin{array}{l}- \\
- \\
- \\
- \\
- \\
-\end{array}$ & $\begin{array}{l}- \\
- \\
- \\
- \\
-\end{array}$ & $\begin{array}{l}- \\
- \\
- \\
- \\
- \\
-\end{array}$ & $\begin{array}{l}10000 \\
19999 \\
19999 \\
24999 \\
24999 \\
19998\end{array}$ & $\begin{array}{ll}7 & 356 \\
7 & 219 \\
8 & 413 \\
8 & 462 \\
8 & 049 \\
7 & 931\end{array}$ & $\begin{array}{l}- \\
- \\
- \\
- \\
- \\
-\end{array}$ & $\begin{array}{l}43402 \\
44020 \\
40835 \\
34574 \\
33696 \\
28687\end{array}$ & $\begin{array}{l}50758 \\
51239 \\
49248 \\
43036 \\
41745 \\
36617\end{array}$ \\
\hline $\begin{array}{l}\text { Jul } \\
\text { Aug } \\
\text { Sep } \\
\text { Oct } \\
\text { Nov } \\
\text { Dec }\end{array}$ & $\begin{array}{r}27943 \\
29126 \\
50847 \\
52568 \\
- \\
-\end{array}$ & $\begin{array}{r}- \\
- \\
- \\
860 \\
- \\
-\end{array}$ & $\begin{array}{r}- \\
- \\
- \\
3700 \\
- \\
-\end{array}$ & $\begin{array}{l}4413 \\
4576 \\
4669 \\
4702 \\
5274 \\
5534\end{array}$ & $\begin{array}{r}2280 \\
22280 \\
2280 \\
2280 \\
\quad- \\
\quad-\end{array}$ & $\begin{array}{r}13892 \\
14974 \\
17646 \\
176849 \\
150812 \\
87410\end{array}$ & $\begin{array}{l}- \\
- \\
- \\
- \\
- \\
-\end{array}$ & $\begin{array}{r}4146 \\
6477 \\
35472 \\
7 \\
7082 \\
7257\end{array}$ & $\begin{array}{rr} & - \\
6477 \\
15056 \\
- \\
- \\
-\end{array}$ & $\begin{array}{r}4146 \\
- \\
20416 \\
7082 \\
7257\end{array}$ & $\begin{array}{r}9999 \\
9999 \\
10000 \\
92239 \\
134768 \\
143273\end{array}$ & $\begin{array}{ll}7 & 714 \\
7 & 871 \\
7 & 907 \\
6 & 712 \\
7 & 082 \\
7 & 257\end{array}$ & $\begin{array}{l}- \\
- \\
- \\
- \\
- \\
-\end{array}$ & $\begin{array}{r}26669 \\
26609 \\
22063 \\
161859 \\
105936 \\
60120\end{array}$ & $\begin{array}{r}38529 \\
40957 \\
65442 \\
168571 \\
120100 \\
74634\end{array}$ \\
\hline
\end{tabular}


Banking, insurance

22 Value of inter-bank clearings

2. United Kingdom

\begin{tabular}{|c|c|c|c|c|c|c|c|c|c|c|c|c|}
\hline & & 1998 & 1999 & 2000 & 2001 & 2002 & 2003 & 2004 & 2005 & 2006 & 2007 & 2008 \\
\hline \multicolumn{13}{|l|}{ Bulk paper clearings ${ }^{1}$} \\
\hline Cheque (formerly general) & KCYY & 1214 & 1226 & 1214 & 1210 & 1178 & 1141 & 1111 & 1062 & 1076 & 1157 & 1076 \\
\hline Credit & KCYZ & 93 & 88 & 82 & 80 & 75 & 69 & 63 & 57 & 56 & 58 & 52 \\
\hline \multicolumn{13}{|l|}{ High-value clearings } \\
\hline Town & KCZA & - & - & - & - & - & - & - & - & .. & & \\
\hline CHAPS Sterling only & KCZB & 41501 & 44704 & 49146 & 52913 & 51896 & 51613 & 52348 & 52672 & 59437 & 69352 & 73626 \\
\hline Electronic clearing (BACS) & KCZC & 1602 & 1761 & 1922 & 2166 & 2382 & 2574 & 2883 & 3150 & 3429 & 3696 & 3946 \\
\hline
\end{tabular}

1 Excludes inter-branch clearings and clearings in Scotland and Northern

Source: APACS - The UK payments association: 02077116223 Ireland. 
$£$ million

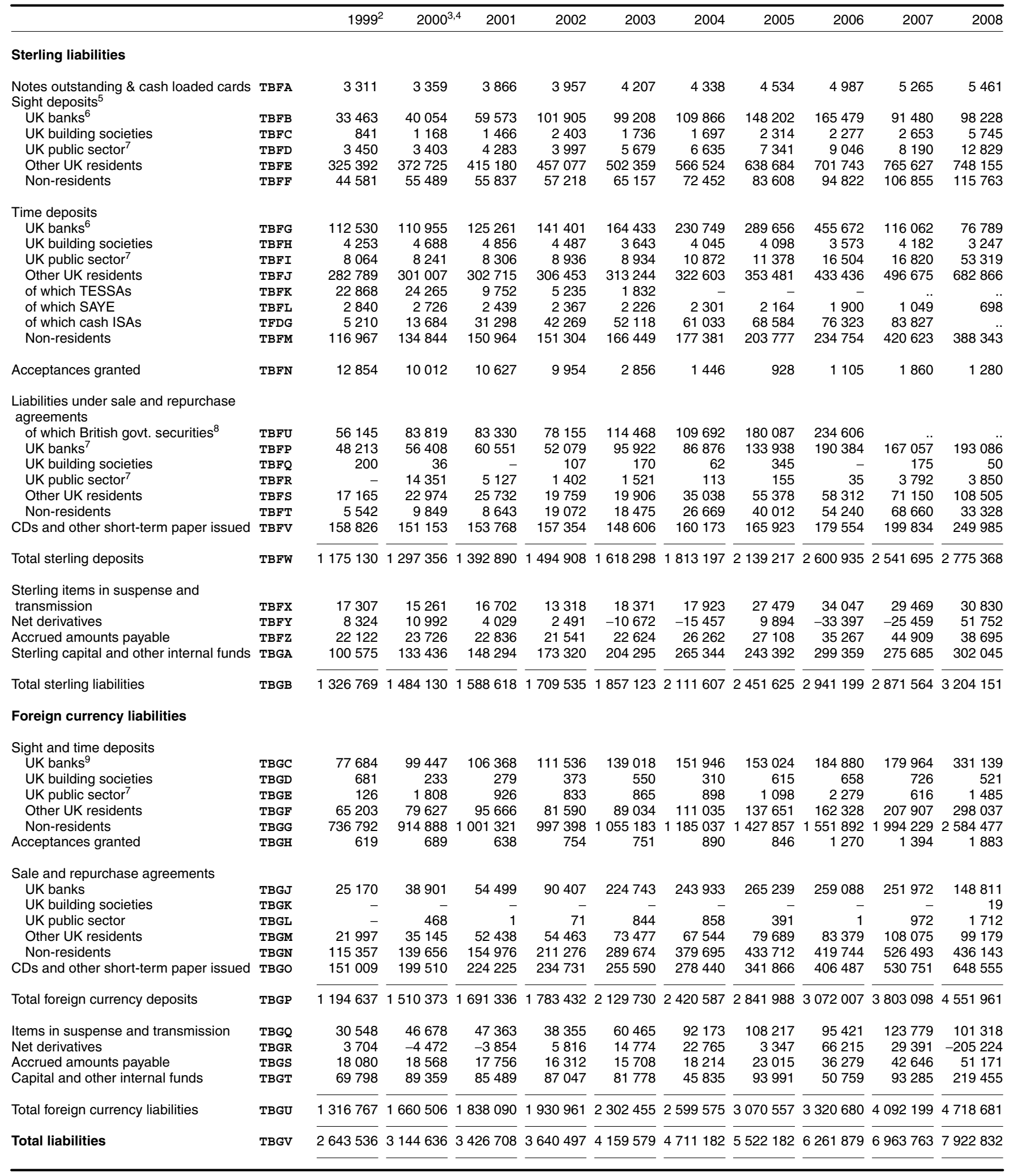


Banking, insurance

\section{Other banks' balance sheet ${ }^{1}$}

\begin{tabular}{|c|c|c|c|c|c|c|c|c|c|c|c|}
\hline \multirow[t]{2}{*}{ continued } & \multirow{2}{*}{\multicolumn{2}{|c|}{$1999^{2}$}} & \multirow[b]{2}{*}{$2000^{3,4}$} & \multirow[b]{2}{*}{2001} & \multirow[b]{2}{*}{2002} & \multirow[b]{2}{*}{2003} & \multirow[b]{2}{*}{2004} & \multirow[b]{2}{*}{2005} & \multicolumn{3}{|r|}{$£$ million } \\
\hline & & & & & & & & & 2006 & 2007 & 2008 \\
\hline \multicolumn{12}{|l|}{ Sterling assets } \\
\hline Notes and coins & TBGW & 9047 & 8007 & 6566 & 6621 & 7464 & 10559 & 9500 & 9279 & 7798 & 7183 \\
\hline \multicolumn{12}{|l|}{ With UK central bank } \\
\hline Cash ratio deposits & TBGX & 1141 & 1275 & 1386 & 1495 & 1609 & 1759 & 1953 & 2271 & 2599 & 2144 \\
\hline Other & TBGY & 676 & 117 & 143 & 249 & 54 & 100 & 1388 & 17645 & 21268 & 51017 \\
\hline \multicolumn{12}{|l|}{ Market loans } \\
\hline UK bank CDs & TBHB & 75071 & 65156 & 68868 & 68728 & 55053 & 59505 & 58780 & 63236 & 50856 & 48696 \\
\hline UK bank commercial paper & TBHC & 208 & 8 & 52 & 62 & 5 & 54 & 75 & 410 & 399 & 783 \\
\hline UK building societies CDs etc and deposits & TBHD & 5093 & 4748 & 3933 & 4293 & 7200 & 7222 & 5655 & 7481 & 7827 & 3673 \\
\hline Non-residents & TBHE & 74403 & 94381 & 102404 & 89848 & 109665 & 102286 & 128343 & 166599 & 268221 & 241265 \\
\hline \multicolumn{12}{|l|}{ Acceptances granted } \\
\hline UK building societies & TBHF & - & - & - & - & - & - & - & - & - & - \\
\hline UK public sector ${ }^{7}$ & TBHG & - & - & - & - & - & - & - & - & - & - \\
\hline Other UK residents & TBHH & 11933 & 9496 & 9992 & 9111 & 2777 & 1348 & 817 & 956 & 1032 & 664 \\
\hline Non-residents & TBHI & 920 & 516 & 635 & 842 & 79 & 99 & 111 & 148 & 828 & 615 \\
\hline \multicolumn{12}{|l|}{ Bills } \\
\hline Treasury bills & TBHJ & 2749 & 1612 & 8474 & 18752 & 18265 & 14507 & 15707 & 11984 & 4837 & 2762 \\
\hline UK bank bills & TBHA & 11426 & 7011 & 8098 & 8491 & 1265 & 646 & 24 & 103 & 51 & 56586 \\
\hline UK building societies & TBHK & - & - & - & - & - & - & - & 375 & - & - \\
\hline Other UK & TBHL & 818 & 1202 & 1601 & 485 & 1013 & 955 & 779 & 1636 & 320 & 421 \\
\hline Non-residents & TBHM & 206 & 287 & 744 & 979 & 733 & 702 & 1063 & 1831 & 4099 & 2622 \\
\hline \multicolumn{12}{|l|}{ Claims under sale and repurchase agreements } \\
\hline of which British govt. securities ${ }^{8}$ & TBHT & 64943 & 86362 & 84068 & 77460 & 114091 & 116652 & 187606 & 237177 & & \\
\hline UK banks & TВHO & 39667 & 46088 & 46585 & 37197 & 77691 & 64353 & 113540 & 160380 & 140814 & 78228 \\
\hline UK building societies & TBHP & 91 & 116 & 327 & 86 & 114 & 263 & 1048 & 76 & 4650 & 4607 \\
\hline UK public sector & TBHQ & - & 9067 & 4692 & 5159 & 5231 & 10801 & 11695 & 9168 & 8025 & 1506 \\
\hline Other UK residents & TBHR & 30338 & 35058 & 36222 & 31363 & 35885 & 50371 & 61311 & 65569 & 102677 & 125017 \\
\hline Non-residents & TBHS & 6310 & 7266 & 7010 & 14271 & 12981 & 20456 & 36261 & 52300 & 68570 & 41216 \\
\hline \multicolumn{12}{|l|}{ Advances } \\
\hline UK public sector & TBHU & 2567 & 2746 & 2442 & 3783 & 4414 & 6078 & 6838 & 8092 & 9443 & 10224 \\
\hline Other UK residents ${ }^{10}$ & TBHV & 732649 & 823787 & 891790 & 986835 & 1062650 & 1159833 & 1252836 & 1376190 & 1555938 & 1665414 \\
\hline Non-residents & TBHW & 23364 & 24494 & 29483 & 31380 & 34603 & 38447 & 49223 & 62314 & 81118 & 86143 \\
\hline Banking dept. lending to central govt. (net) & TBNU & - & - & - & - & - & - & - & .. & .. & .. \\
\hline \multicolumn{12}{|l|}{ Investments } \\
\hline UK banks ${ }^{11}$ & TBHZ & 13584 & 22935 & 23965 & 23542 & 34971 & 34664 & 36365 & 36655 & 37633 & 61700 \\
\hline UK building societies & TBIA & 2506 & 2251 & 2099 & 1835 & 1702 & 1889 & 2068 & 1891 & 1009 & 605 \\
\hline Other UK residents ${ }^{12}$ & TBIB & 57391 & 77647 & 82013 & 76773 & 84813 & 100892 & 112593 & 165898 & 165949 & 265092 \\
\hline Non-residents & TBIC & 13775 & 20572 & 23462 & 22821 & 22505 & 26029 & 30487 & 39000 & 46197 & 44218 \\
\hline Items in suspense and collection & TBID & 23441 & 21982 & 24024 & 19577 & 22434 & 22066 & 29714 & 34629 & 40301 & 48176 \\
\hline Accrued amounts receivable & TBIE & 15173 & 15919 & 13528 & 15486 & 17204 & 19987 & 19510 & 24909 & 30814 & 34711 \\
\hline Other assets & TBIF & 13036 & 12654 & 12876 & 12685 & 11955 & 12875 & 15522 & 14430 & 14749 & 15808 \\
\hline Total sterling assets ${ }^{13}$ & TBIG & 321486 & 4685271 & 595380 & 1727136 & 889200 & 2107562 & 436032 & 2943591 & 2851729 & 3088382 \\
\hline
\end{tabular}




\begin{tabular}{|c|c|c|c|c|c|c|c|c|c|c|c|}
\hline & & $1999^{2}$ & $2000^{3,}$ & 2001 & 2002 & 2003 & 2004 & 2005 & 2006 & 2007 & 2008 \\
\hline \multicolumn{12}{|l|}{ Foreign currency assets } \\
\hline \multicolumn{12}{|l|}{ Market loans and advances } \\
\hline UK banks ${ }^{9}$ & TBIH & 74250 & 93269 & 104107 & 114809 & 137417 & 149119 & 146316 & 173211 & 177957 & 334360 \\
\hline UK banks' CDs etc & TBII & 14364 & 13171 & 13298 & 10128 & 13162 & 11026 & 18412 & 13023 & 12851 & 8635 \\
\hline UK building societies CDs etc. and deposits & TBIJ & 451 & 173 & 354 & 357 & 591 & 448 & 411 & 422 & 608 & 304 \\
\hline UK public sector ${ }^{7}$ & TBIK & 20 & 30 & 13 & 83 & 91 & 50 & 153 & 105 & 153 & 52 \\
\hline Other UK residents & TBIL & 88847 & 107707 & 118106 & 117669 & 134894 & 153574 & 161488 & 199147 & 279617 & 389404 \\
\hline \multicolumn{12}{|l|}{ Claims under sale and repurchase agreement } \\
\hline UK banks & TBIO & 28008 & 41801 & 61188 & 91488 & 225027 & 250209 & 272361 & 257856 & 248960 & 144105 \\
\hline Iding societies & TBIP & - & - & - & - & - & - & 100 & 67 & 2152 & 4434 \\
\hline UK public sector ${ }^{7}$ & TBIQ & - & 737 & 23 & 486 & 1420 & 1590 & 1125 & 1 & 1118 & 413 \\
\hline Other UK residents & TBIR & 33027 & 57876 & 73237 & 86866 & 100817 & 106973 & 135018 & 131709 & 171482 & 160563 \\
\hline Non-residents & TBIS & 146756 & 199990 & 219449 & 256663 & 382672 & 506157 & 612065 & 595615 & 751407 & 539082 \\
\hline Acceptances granted & TBIT & 619 & 689 & 638 & 754 & 751 & 890 & 846 & 1270 & 1394 & 1883 \\
\hline Bills & TBIU & 19508 & 21878 & 25399 & 20803 & 31429 & 24250 & 24230 & 25917 & 35841 & 40709 \\
\hline British government stocks & TBIV & 4473 & 3518 & 890 & 226 & 19 & -20 & -30 & 104 & 9 & 2702 \\
\hline Other public sector & TBIW & - & - & 4 & 18 & 7 & - & - & 1 & - & 1 \\
\hline UK banks & TBIX & 8607 & 11706 & 10633 & 10298 & 11688 & 13187 & 13176 & 12384 & 13811 & 28094 \\
\hline UK building societies & TBIY & 631 & 939 & 850 & 1170 & 1570 & 2400 & 2974 & 2683 & 2590 & 3399 \\
\hline Other UK residents & TBIZ & 5679 & 12298 & 18129 & 20130 & 21846 & 32882 & 45283 & 57858 & 69167 & 102289 \\
\hline Non-residents & TBJA & 243147 & 297404 & 324073 & 326035 & 334371 & 398040 & 484874 & 566025 & 662689 & 599092 \\
\hline Items in suspense & TBJB & 29706 & 44885 & 55026 & 44037 & 60804 & 81937 & 112089 & 117498 & 122409 & 118942 \\
\hline Accrued amounts receivable & TBJC & 20163 & 21279 & 18969 & 19434 & 18129 & 19561 & 22397 & 32639 & 42078 & 60164 \\
\hline Other assets & TBJD & 4648 & 2978 & 3880 & 8735 & 13683 & 11802 & 19089 & 26251 & 23691 & 27957 \\
\hline Total foreign currency assets ${ }^{14}$ & TBJE & 13220501 & 1676109 & 1831322 & 1913355 & 2270372 & 2603610 & 3086141 & 3318281 & 4112034 & 44834450 \\
\hline Total assets & TBJF & 26435363 & 3144636 & 3426702 & 3640491 & 4159572 & 4711173 & 5522173 & 6261872 & 6963763 & 37922832 \\
\hline Holdings of own sterling acceptances & TBJG & 1725 & 1231 & 916 & 1220 & 411 & 265 & 24 & 19 & 20 & 14 \\
\hline Holdings of own FC acceptances & TBJH & 150 & 135 & 118 & 58 & 104 & 170 & 222 & 247 & 526 & 888 \\
\hline Eligible banks' total sterling acceptances & TBJI & 14523 & 10597 & 11320 & 10805 & 3035 & 1217 & 152 & & & \\
\hline Eligible liabilities & TBJJ & 849289 & 952062 & 1012194 & 1087877 & 1163917 & 1266726 & 1420348 & 1636053 & 31873503 & 32086706 \\
\hline
\end{tabular}

1 See chapte

9 Foreign currency sight and time deposits from UK banks and foreign currency

2 Data for 1999 reflect the acquisition of Birmingham Midshires Building

Society by Halifax during that year.

3 Data for 2000 reflect the entry of Bradford \& Bingley plc to the banking sector during the year.

4 Data for 2000 reflect the new reporting during the year of agency business as a result of collateral management via repos and reverse repos. market loans and advances to UK banks in 2001, 2003 and 2004 were each depressed by $£ 14.5 \mathrm{bn}, £ 0.5$ bn and $£ 4.7$ bn respectively as a result of positions being consolidated out on the merger of two banks.

10 During 2000, 2001, 2002, 2003, 2004 and 2005 sterling advances to other UK residents were reduced by $£ 10.3 \mathrm{bn}, £ 12.9 \mathrm{bn}, £ 16.2 \mathrm{bn}, £ 29.1 \mathrm{bn}, £ 30.4 \mathrm{bn}$ and £33.6 bn respectively as a result of securitisations and other loan trans-

5 Sterling sight deposits from UK banks and sterling market loans to UK Sterling investments in UK banks in 2000 were boosted by Barclay's £5.8 bn banks in 2003 were depressed by $£ 19$ bn following the consolidation of two 11 Sterling investments in
banks balance sheets.

6 Sterling deposits (sight plus time) from, and lending to banks in 2007 were 12 Sterling investments in other UK residents in 2000 were boosted by Lloyds reduced by £386bn following changes in the reporting population. TSB's £5.8 bn investment in Scottish Widows Group.

7 From 2000 the UK public sector series reflects assumption by the Debt 13 Changes in the reporting populations in 1998, 1999, 2000, 2001, 2003 and Management Office (an executive agency of HM Treasury) of responsibility 2004 account for a net decrease of $£ 7.3$ bn, £11.3 bn £0.8 bn, £0.7bn, £0.2 bn for government cash management. and £4.5 bn respectively in sterling assets outstanding. 8 Due to changes to the forms completed by reporting institutions, the break- 14 Changes in the reporting populations in 1998, 1999, 2000, 2001 , 2003 and not available after 2006 and $£ 5.2$ bn of foreign currency assets outstanding. 


\section{Industrial analysis of bank lending to UK residents ${ }^{1}$}

$£$ million

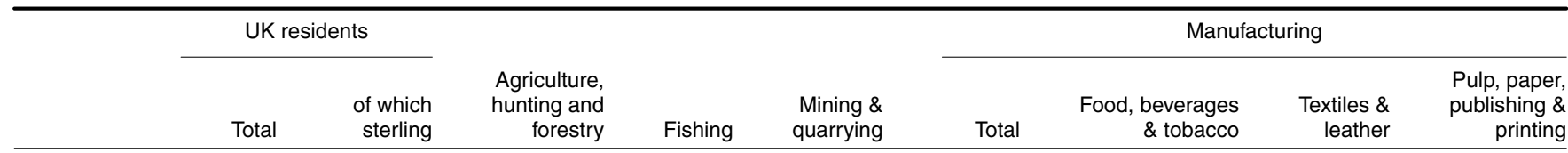

Amounts outstanding (sterling \& other currencies)

Loans \& advances (including under repo \& sterling commercial paper)

\begin{tabular}{ccccc}
\multicolumn{1}{c}{ TBOA } & TBOB & TBOC & TBOD \\
2006 & 1793840 & 1460380 & 9620
\end{tabular}

2007

1793840

1460380

413

quarrying

Total $\&$ tobacco printing

Acceptances

$\begin{array}{lr} & \text { TBQA } \\ 2006 & 1190 \\ 2007 & \\ \text { Total } & \\ & \text { TBSA } \\ 2007 & 212845 \\ 2008 & 262962\end{array}$

of which in sterling

$\begin{array}{ll}2007 & \text { TBUA } \\ 2008 & 267608 \\ & 2077692\end{array}$

Facilities granted

$\begin{array}{lr} & \text { TCAA } \\ 2007 & 2594187 \\ 2008 & 309800\end{array}$

of which in sterling

\begin{tabular}{rrr} 
& TCCA & \\
2007 & 1977125 & \\
2008 & & \\
\hline & $\begin{array}{r}\text { Chemicals, } \\
\text { man-made fibres, } \\
\text { rubber \& } \\
\text { plastics }\end{array}$ & $\begin{array}{r}\text { Non-metallic } \\
\text { mineral products } \\
\text { \& metals }\end{array}$ \\
& & \\
\hline
\end{tabular}

TBQC TBQD

TBQB
956

$-$

$-$

$\begin{array}{rr}\text { TBQE } & \text { TBQF } \\ 1 & 104\end{array}$

Amounts outstanding (sterling \& other currencies)

\begin{tabular}{|c|c|c|c|c|c|c|c|c|}
\hline \multicolumn{9}{|c|}{ Loans \& advances (including under repo \& sterling commercial paper) } \\
\hline & TBOJ & TBOK & TBOL & TBOM & TBON & TBOO & TBOP & TBOQ \\
\hline 2006 & 5681 & 6122 & 6678 & 3741 & 5903 & 7075 & 4235 & 20671 \\
\hline 2007 & .. & .. & .. & .. & .. & - & .. & .. \\
\hline Acce & & & & & & & & \\
\hline & TBQJ & TBQK & TBQL & TBQM & TBQN & TBQO & TBQP & TBQQ \\
\hline 2006 & 4 & 10 & 8 & 14 & 33 & - & - & 15 \\
\hline 2007 & .. & .. & .. & .. & .. & - & .. & \\
\hline Total & & & & & & & & \\
\hline & TBSJ & TBSK & TBSL & TBSM & TBSN & TBSO & TBSP & TBSQ \\
\hline 2007 & .. & 6594 & 7461 & .. & 6482 & 6485 & 2449 & 27187 \\
\hline 2008 & .. & 8036 & 10605 & .. & 6148 & 9765 & 3728 & 31165 \\
\hline of $w$ & & & & & & & & \\
\hline & TBUJ & TBUK & TBUL & TBUM & TBUN & TBUO & TBUP & TBUQ \\
\hline 2007 & 2864 & 3842 & 3820 & 1994 & 5103 & 5559 & 2435 & 26062 \\
\hline 2008 & 3191 & 3703 & 4968 & 2127 & 4017 & 7605 & 3697 & 30087 \\
\hline Facili & & & & & & & & \\
\hline 2008 & .. & 12928 & 18231 & 9003 & 11498 & 18712 & 8042 & 42884 \\
\hline of $\mathbf{w}$ & & & & & & & & \\
\hline & TCCJ & TCCK & TCCL & TCCM & TCCN & TCCO & TCCP & TCCQ \\
\hline 2007 & 5160 & 6640 & 7527 & 3380 & 7618 & 9064 & 5628 & 37118 \\
\hline 2008 & 4760 & 6113 & 8847 & 3 464 & 5746 & 11825 & 7184 & 40030 \\
\hline
\end{tabular}




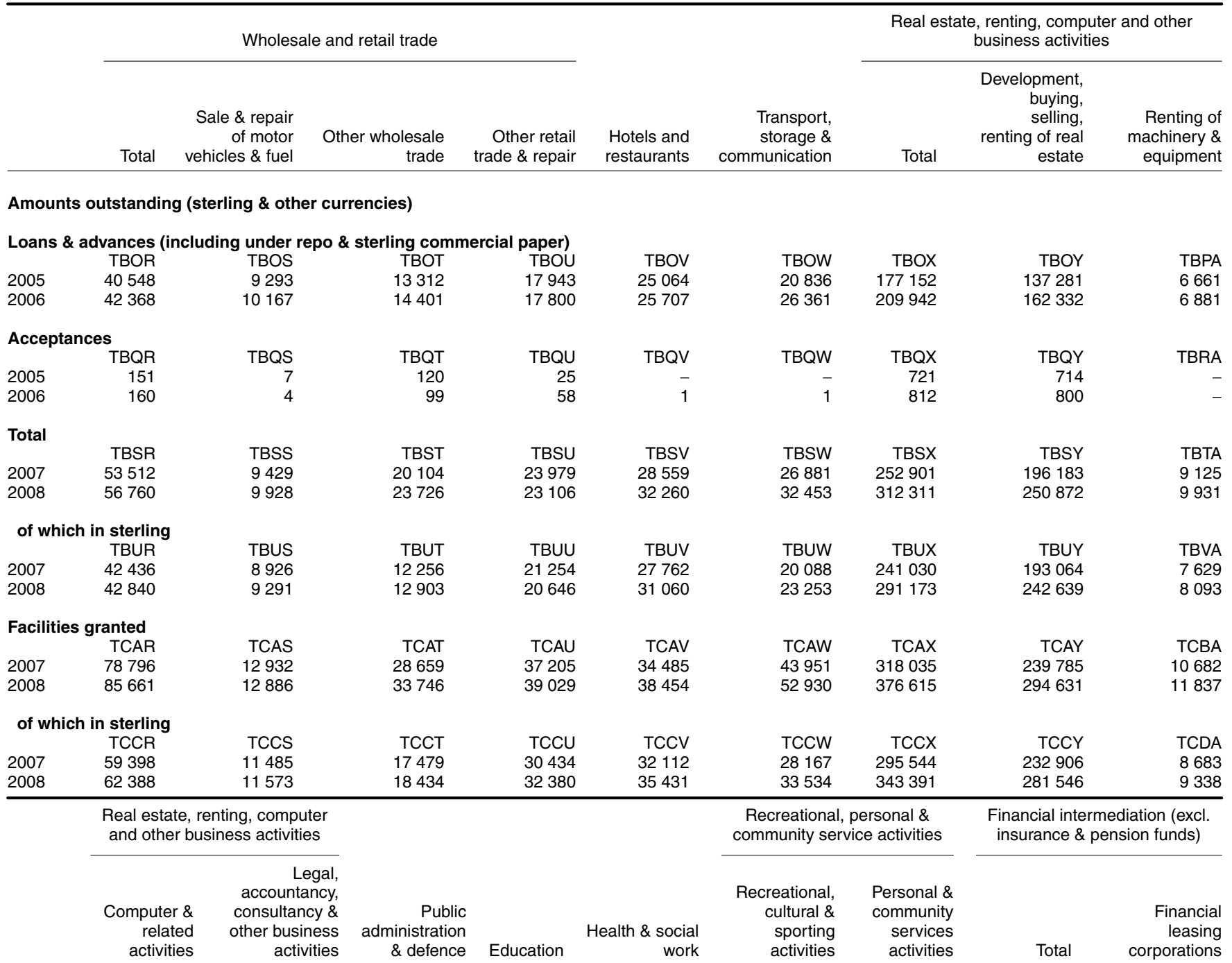

Amounts outstanding (sterling \& other currencies)

\begin{tabular}{|c|c|c|c|c|c|c|c|c|c|}
\hline \multicolumn{10}{|c|}{ Loans \& advances (including under repo \& sterling commercial paper) } \\
\hline & TBPB & TBPC & TBPD & TBPE & TBPF & TBPH & TBPG & TBPI & TBPJ \\
\hline 2006 & 4431 & 36299 & 17227 & 7498 & 15854 & 12255 & 5594 & 491121 & 41068 \\
\hline 2007 & .. & .. &.. & .. & .. & .. & .. & - & .. \\
\hline Acce & & & & & & & & & \\
\hline & TBRB & TBRC & TBRD & TBRE & TBRF & TBRH & TBRG & TBRI & TBRJ \\
\hline 2006 & - & 12 & - & - & - & - & 5 & 90 & - \\
\hline 2007 & .. & .. & .. & .. & .. & .. & .. & - & .. \\
\hline Total & & & & & & & & & \\
\hline & TBTB & TBTC & TBTD & TBTE & TBTF & TBTH & TBTG & TBTI & TBTJ \\
\hline 2007 & 4590 & 43004 & 18539 & 8720 & 19233 & 14308 & 7038 & 662127 & 42290 \\
\hline 2008 & 5405 & 46103 & 31792 & 10533 & 21434 & 14245 & 7023 & 832718 & 46518 \\
\hline of $w$ & ling & & & & & & & & \\
\hline & TBVB & TBVC & TBVD & TBVE & TBVF & TBVH & TBVG & TBVI & TBVJ \\
\hline 2007 & 2829 & 37508 & 17336 & 8626 & 18808 & 13273 & 6420 & 353327 & 35470 \\
\hline 2008 & 2813 & 37629 & 31282 & 10347 & 20945 & 12722 & 6232 & 489589 & 35862 \\
\hline Facili & & & & & & & & & \\
\hline 2007 & 7411 & 60156 & 21493 & 11789 & 22910 & 19589 & 9403 & 750335 & 44850 \\
\hline 2008 & 7703 & 62443 & 35094 & 13871 & 24582 & 19977 & 9293 & 920092 & 50169 \\
\hline of $w$ & ling & & & & & & & & \\
\hline & TCDB & TCDC & TCDD & TCDE & TCDF & $\mathrm{TCDH}$ & TCDG & TCDI & TCDJ \\
\hline 2007 & 4431 & 49524 & 20263 & 11540 & 22099 & 17088 & 8395 & 383376 & 37676 \\
\hline 2008 & 3892 & 48614 & 33737 & 13431 & 23710 & 16416 & 8012 & 515630 & 39211 \\
\hline
\end{tabular}




\begin{tabular}{|c|c|c|c|c|c|c|c|c|c|}
\hline & \multicolumn{9}{|c|}{ Financial intermediation (excl. insurance \& pension funds) } \\
\hline & $\begin{array}{l}\text { Non-bank credit } \\
\text { grantors, excl. } \\
\text { credit unions }\end{array}$ & Credit unions & $\begin{array}{r}\text { Factoring } \\
\text { corporations }\end{array}$ & $\begin{array}{r}\text { Mortgage \& } \\
\text { housing credit } \\
\text { corporations }\end{array}$ & $\begin{array}{r}\text { Investment \& } \\
\text { unit trusts } \\
\text { excl. money } \\
\text { market mutual } \\
\text { funds }\end{array}$ & $\begin{array}{l}\text { Money market } \\
\text { mutual funds }\end{array}$ & $\begin{array}{r}\text { Bank holding } \\
\text { companies }\end{array}$ & $\begin{array}{l}\text { Securities } \\
\text { dealers }(f)\end{array}$ & $\begin{array}{l}\text { Other financial } \\
\text { intermediaries }\end{array}$ \\
\hline \multicolumn{10}{|c|}{ Amounts outstanding (sterling \& other currencies) } \\
\hline \multicolumn{10}{|c|}{ Loans \& advances (including under repo \& sterling commercial paper) } \\
\hline & TBPK & TBPL & TBPM & TBPN & TBPO & TBPP & TBPQ & TBPR & TBPS \\
\hline 2005 & 17833 & 28 & 4633 & 62869 & 20394 & 1377 & 19707 & 165421 & 93916 \\
\hline 2006 & 21496 & 60 & 5593 & 84959 & 20131 & 674 & 17969 & 183551 & 115620 \\
\hline \multicolumn{10}{|c|}{ Acceptances } \\
\hline & TBRK & TBRL & TBRM & TBRN & TBRO & TBRP & TBRQ & TBRR & TBRS \\
\hline 2005 & 15 & 5 & - & - & - & - & - & - & 27 \\
\hline 2006 & 15 & 5 & - & - & - & - & - & - & 69 \\
\hline \multicolumn{10}{|l|}{ Total } \\
\hline & TBTK & TBTL & TBTM & TBTN & ТВTO & TBTP & TBTQ & TBTR & TBTS \\
\hline 2007 & 23682 & 80 & 6524 & 99231 & 20980 & 327 & 44392 & 218224 & 206398 \\
\hline 2008 & 25167 & 83 & 7072 & 176722 & 11583 & 394 & 53453 & 200244 & 311483 \\
\hline \multicolumn{10}{|c|}{ of which in sterling } \\
\hline & TBVK & TBVL & TBVM & TBVN & TBVO & TBVP & TBVQ & TBVR & TBVS \\
\hline 2007 & 21443 & 80 & 5520 & 82577 & 6133 & 77 & 32363 & 36975 & 132688 \\
\hline 2008 & 21919 & 82 & 5742 & 147755 & 6700 & 29 & 38476 & 33451 & 199573 \\
\hline \multicolumn{10}{|c|}{ Facilities granted } \\
\hline & TCBK & TCBL & TCBM & TCBN & TCBO & TCBP & $\mathrm{TCBQ}$ & TCBR & TCBS \\
\hline 2007 & 25847 & 93 & 6857 & 105225 & 48565 & 400 & 45288 & 232192 & 241017 \\
\hline 2008 & 27248 & 95 & 7367 & 179845 & 44006 & 481 & 56075 & 206462 & 348344 \\
\hline \multicolumn{10}{|c|}{ of which in sterling } \\
\hline & TCDK & TCDL & TCDM & TCDN & TCDO & TCDP & $\mathrm{TCDQ}$ & TCDR & TCDS \\
\hline 2007 & 23119 & 90 & 5786 & 88024 & 12915 & 89 & 32504 & 37961 & 145212 \\
\hline 2008 & 23378 & 94 & 5965 & 150263 & 12972 & 29 & 39367 & 33994 & 210358 \\
\hline \multicolumn{6}{|c|}{$\begin{array}{l}\text { Activities auxiliary to financial } \\
\text { intermediation }\end{array}$} & \multicolumn{4}{|c|}{ Individuals \& individual trusts } \\
\hline & Insurar & $\begin{array}{r}\text { ce companies \& } \\
\text { pension funds }\end{array}$ & Fund $n$ & $\begin{array}{r}\text { tanagement } \\
\text { activities }\end{array}$ & Other & Total & $\begin{array}{r}\text { Lending se } \\
\text { dwell } \\
\text { bridging }\end{array}$ & $\begin{array}{l}\text { cured on } \\
\text { llings inc. } \\
\text { g finance }\end{array}$ & $\begin{array}{r}\text { Other loans \& } \\
\text { advances }\end{array}$ \\
\hline \multicolumn{10}{|c|}{ Amounts outstanding (sterling \& other currencies) } \\
\hline \multicolumn{10}{|c|}{ Loans \& advances (including under repo \& sterling commercial paper) } \\
\hline & & TBPT & & TBPU & TBPV & TBPW & & TBPX & TBPY \\
\hline 2007 & & .. & & .. & .. & 749174 & & 605198 & 144014 \\
\hline 2008 & &.. & &.. &.. & 896809 & & 755982 & 140827 \\
\hline \multicolumn{10}{|c|}{ Acceptances } \\
\hline & & TBRT & & TBRU & TBRV & & & & \\
\hline 2006 & & 1 & & - & - & & & & \\
\hline 2007 & & .. & & - &.. & & & & \\
\hline \multicolumn{10}{|l|}{ Total } \\
\hline & & TBTT & & TBTU & TBTV & TBTW & & TBTX & TBTY \\
\hline 2007 & & 30306 & & 52748 & 93324 & 749174 & & 605198 & 144014 \\
\hline 2008 & & 30681 & & 34604 & 179106 & 896809 & & 755982 & 140827 \\
\hline \multicolumn{10}{|c|}{ of which in sterling } \\
\hline & & TBVT & & TBVU & TBVV & TBVW & & TBVX & TBVY \\
\hline 2007 & & 27965 & & 15774 & 58154 & 747490 & & .. & 142853 \\
\hline 2008 & & 25678 & & 14089 & 97578 & 894205 & &.. & 139269 \\
\hline \multicolumn{10}{|c|}{ Facilities granted } \\
\hline & & TCBT & & TCBU & TCBV & TCBW & & TCBX & TCBY \\
\hline 2007 & & 58775 & & 56988 & 96108 & 865188 & & 667425 & 197762 \\
\hline 2008 & & 57474 & & 44484 & 182701 & 1013900 & & 812818 & 201082 \\
\hline \multicolumn{10}{|c|}{ of which in sterling } \\
\hline & & TCDT & & TCDU & TCDV & TCDW & & TCDX & TCDY \\
\hline 2007 & & 38374 & & 17865 & 59923 & 863182 & & 666897 & 142853 \\
\hline 2008 & & 30977 & & 15207 & 99601 & 1010884 & & 811743 & 199141 \\
\hline
\end{tabular}

1 See chapter text. 


\section{5 Industrial analysis of bank deposits from UK residents ${ }^{1}$}

$£$ million

\begin{tabular}{|c|c|c|c|c|c|c|c|c|}
\hline & \multirow[b]{2}{*}{$\begin{array}{r}\text { Total from UK } \\
\text { residents }\end{array}$} & \multirow[b]{2}{*}{$\begin{array}{r}\text { Agriculture, } \\
\text { hunting and } \\
\text { forestry }\end{array}$} & \multirow[b]{2}{*}{ Fishing } & \multirow[b]{2}{*}{$\begin{array}{r}\text { Mining \& } \\
\text { quarrying }\end{array}$} & \multicolumn{4}{|c|}{ Manufacturing } \\
\hline & & & & & Total & $\begin{array}{r}\text { Food, beverages } \\
\& \text { tobacco }\end{array}$ & $\begin{array}{l}\text { Textiles \& } \\
\text { leather }\end{array}$ & $\begin{array}{r}\text { Pulp, paper, } \\
\text { publishing \& } \\
\text { printing }\end{array}$ \\
\hline \multicolumn{9}{|c|}{ Amounts outstanding (sterling \& other currencies) } \\
\hline \multicolumn{9}{|c|}{ Deposit liabilities (including under repos) } \\
\hline & TDAA & TDAB & TDAC & TDAD & TDAE & TDAF & TDAG & TDAH \\
\hline 2008 & 2293013 & 5265 & 175 & 10582 & 38880 & 3594 & 1121 & 3737 \\
\hline \multicolumn{9}{|c|}{ of which in sterling } \\
\hline & TDCA & TDCB & TDCC & TDCD & TDCE & TDCF & TDCG & $\mathrm{TDCH}$ \\
\hline 2007 & 1362254 & 4969 & 176 & 3149 & 30692 & 2738 & 1042 & 4246 \\
\hline \multirow[t]{2}{*}{2008} & 1889330 & 5024 & 162 & 2868 & 28101 & 2370 & 850 & 3030 \\
\hline & \multicolumn{5}{|c|}{ Manufacturing } & \multicolumn{2}{|c|}{ Electricity, gas and water supply } & \\
\hline
\end{tabular}

Amounts outstanding (sterling \& other currencies)

Deposit liabilities (including under repos)

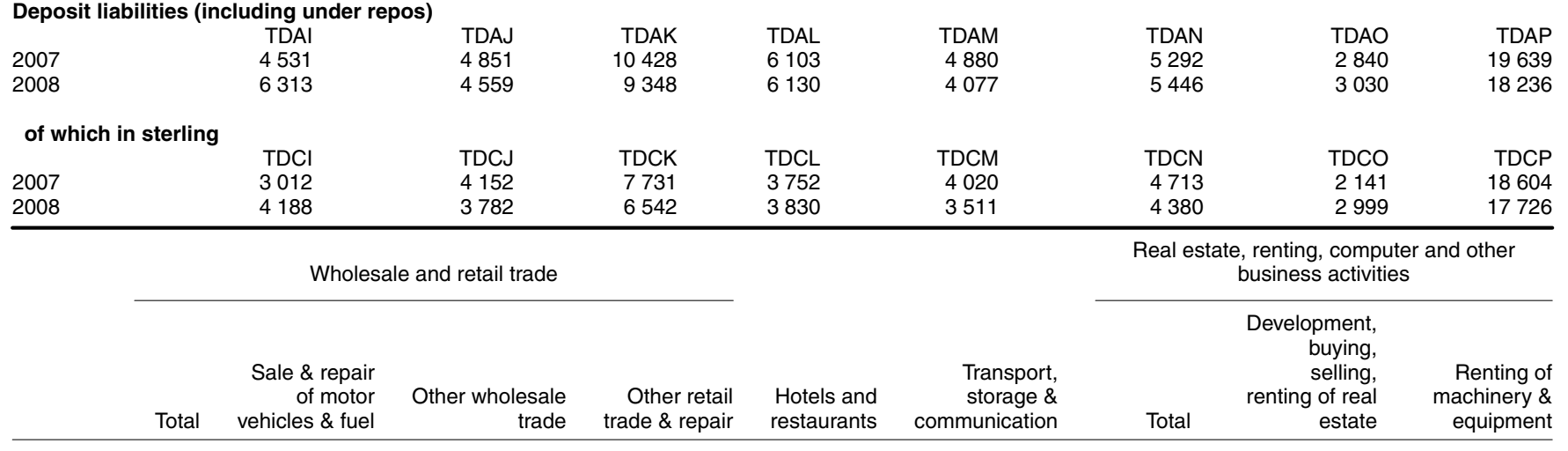

Amounts outstanding (sterling \& other currencies)

Deposit liabilities (including under repos)

\begin{tabular}{|c|c|c|c|c|c|c|c|c|c|}
\hline \multicolumn{10}{|c|}{ TDAD TDAB } \\
\hline & TDAQ & TDAR & TDAS & TDAT & TDAU & TDAV & TDAW & TDAX & TDAY \\
\hline 2007 & 32478 & 4008 & 14592 & 13878 & 5339 & 20753 & 123626 & 36580 & 1623 \\
\hline 2008 & 30492 & 4063 & 13461 & 12968 & 4380 & 19345 & 121518 & 33599 & 1750 \\
\hline \multicolumn{10}{|c|}{ of which in sterling } \\
\hline 2007 & 27713 & 3558 & 11110 & 13045 & 5042 & 16350 & 113982 & 35531 & 1419 \\
\hline 2008 & 25856 & 3561 & 10288 & 12007 & 4198 & 15075 & 109550 & 32623 & 1564 \\
\hline
\end{tabular}


Banking, insurance

\section{Industrial analysis of bank deposits from UK residents

Real estate, renting, computer

and other business activities

Legal,

Computer \& consultancy \&

other business

activities activities

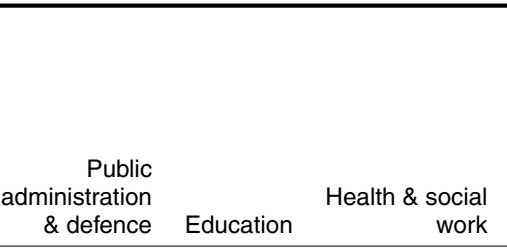

Recreational, personal \&

community service activities

Financial intermediation (excl.

insurance \& pension funds)

Recreational, Personal \&

cultural \& community

sporting services activities activities

Financial

leasing Total corporations

Amounts outstanding (sterling \& other currencies)

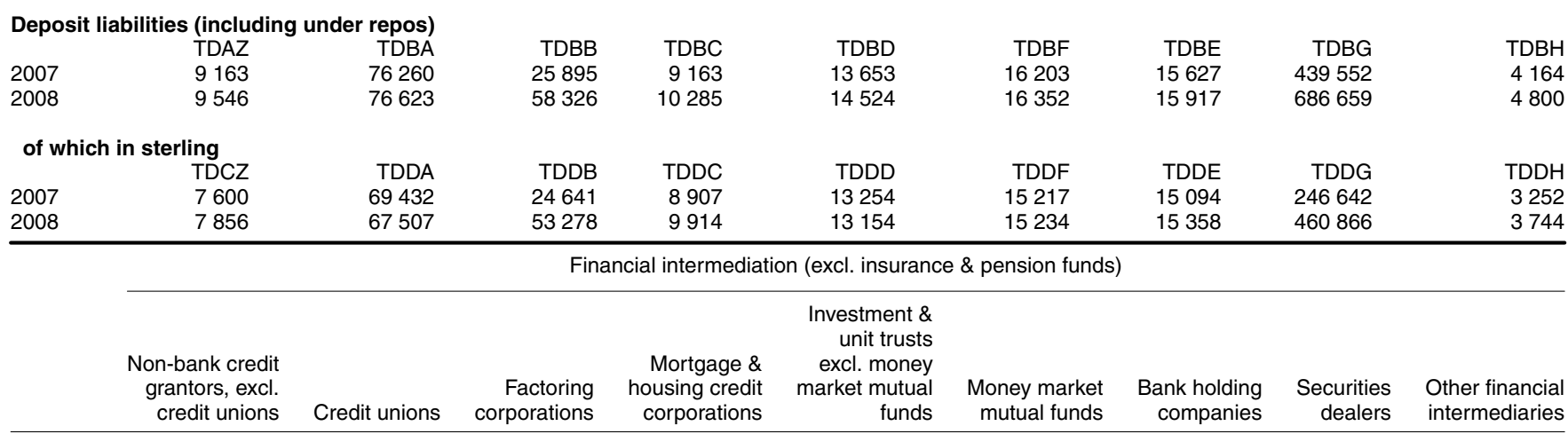

Amounts outstanding (sterling \& other currencies)

Deposit liabilities (including under repos)

\begin{tabular}{|c|c|c|c|c|c|c|c|c|c|}
\hline \multicolumn{10}{|c|}{ 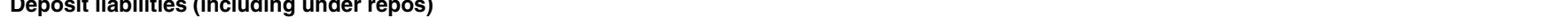 } \\
\hline 管 & TDBI & TDBJ & TDBK & TDBL & TDBM & TDBN & TDBO & TDBP & TDBQ \\
\hline 2007 & 9557 & 438 & 768 & 12887 & 36033 & 489 & 39870 & 154352 & 180996 \\
\hline 2008 & 8818 & 443 & 829 & 110840 & 41004 & 377 & 64918 & 143430 & 311200 \\
\hline \multicolumn{10}{|c|}{ of which in sterling } \\
\hline & TDDI & TDDJ & TDDK & TDDL & TDDM & TDDN & TDDO & TDDP & TDD \\
\hline 2007 & 7753 & 438 & 631 & 12601 & 19093 & 358 & 27013 & 41855 & 13364 \\
\hline \multirow[t]{3}{*}{2008} & 6455 & 443 & 678 & 108814 & 23247 & 233 & 45040 & 35604 & 236610 \\
\hline & \multirow{2}{*}{\multicolumn{3}{|c|}{$\begin{array}{r}\text { Insurance companies \& pension } \\
\text { funds }\end{array}$}} & \multicolumn{6}{|c|}{ Activities auxiliary to financial intermediation } \\
\hline & & & & Place & managers & & & \multicolumn{2}{|c|}{ Individuals \& individual trusts } \\
\hline
\end{tabular}

Amounts outstanding (sterling \& other currencies)

Deposit liabilities (including under repos)

\begin{tabular}{lr}
2007 & TDB \\
2008 & 68820 \\
& 65941 \\
of which in sterling & \\
2007 & TDD \\
2008 & 6024 \\
\hline
\end{tabular}

1 See chapter text. 
23.6 Public sector net cash requirement and other counterparts to changes in money

\begin{tabular}{|c|c|c|c|c|c|c|c|c|c|c|c|c|}
\hline \multirow{2}{*}{\multicolumn{2}{|c|}{ Not seasonally adjusted }} & \multicolumn{11}{|r|}{$£$ million } \\
\hline & & 1998 & 1999 & 2000 & 2001 & 2002 & 2003 & 2004 & 2005 & 2006 & 2007 & 2008 \\
\hline $\begin{array}{l}\text { ublic sector net cash requirement } \\
\text { surplus) }\end{array}$ & ABEN & -6296 & -3205 & -36864 & -2019 & 18010 & 37160 & 41915 & 41278 & 33916 & 32362 & 79456 \\
\hline les of public sector debt to M4 private sector & IDH8 & 1698 & -1448 & 13639 & 7716 & -9258 & -32438 & -32007 & -11257 & -20082 & -16293 & \\
\hline 4 lending ${ }^{1}$ & AVBS & 63926 & 78029 & 111202 & 82574 & 107553 & 127820 & 156084 & 158087 & 218445 & 238495 & 269792 \\
\hline oney stock (M4) & AUZI & 60097 & 33329 & 67198 & 58994 & 68834 & 73271 & 100014 & 150869 & 167008 & 181535 & 270845 \\
\hline
\end{tabular}

1 Bank and building society lending, plus holdings of commercial bills by the

Source: Bank of England: 02076015468

Issue Department of the Bank of England.

2 Including sterling lending to non-residents sector.

\subsection{Money stock and liquidity $^{2}$}

$£$ million

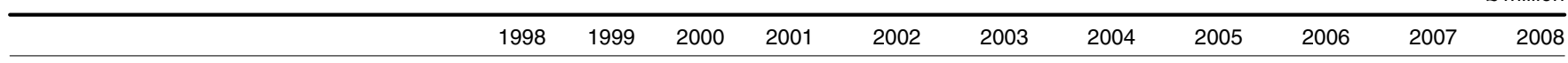

Amounts outstanding at end-year

Notes and coin in circulation with the

M4 private sector ${ }^{1}$
UK private sector sterling non-interest

bearing sight deposits ${ }^{2}$ non-interest

Money stock (M2) ${ }^{3}$

VQKT

AUYA

$23705 \quad 26269 \quad 28174 \quad 30450$

$31889 \quad 34010 \quad 36410$

38508

40523

43567

44868

roxv

$36765 \quad 42130 \quad 45867 \quad 50548$

$45594 \quad 51274 \quad 50845$

$55208 \quad 54800$

62051

70114

Money stock M4

AUYM 7833548166018848739425941008750108129911791921328321149892016748551949837

Changes during the year ${ }^{4}$

Notes and coin in circulation with the

M4 private sector ${ }^{1}$.

UK private sector sterling non-interest

bearing sight deposits ${ }^{2}$

\begin{tabular}{lrrrrrrrrrrr} 
VQLU & 1501 & 2582 & 1957 & 2284 & 1493 & 2189 & 2461 & 2156 & 2037 & 3116 & 1265 \\
AUZA & -753 & 5354 & 3534 & 4913 & -6760 & 5322 & -227 & 5701 & -408 & 9292 & -1964 \\
AUZE & 30781 & 41992 & 39123 & 52813 & 53698 & 72255 & 68901 & 78428 & 72764 & 65042 & 48558 \\
AUZI & 60097 & 33329 & 67198 & 58994 & 68834 & 73271 & 100014 & 150869 & 167008 & 181535 & 270845 \\
\hline
\end{tabular}

Money stock (M2) ${ }^{3}$

AUZI $\quad 60097 \quad 33329 \quad 67198 \quad 58994$

$\begin{aligned} & 1 \text { The estimates of levels of coin in circulation include allowance for wastage, } 3 \text { M2 comprises the UK non-monetary financial institutions and non-public sec- } \\ & \text { hoarding, etc. }\end{aligned}$
tor, i.e. M4 private sector's holdings of notes and coin together with its sterling hoarding, etc. denominated retail deposits with UK monetary financial institutions.

ed in the United Kingdom banks sector (See Table 23.3).

4 As far as possible the changes exclude the effect of changes in the number of contributors to the series, and also of the introduction of new statistical returns. Changes are not seasonally adjusted. 
Banking, insurance

Selected retail banks' base rate ${ }^{1}$

Operative between dates shown

Percentage rates

\begin{tabular}{|c|c|c|c|c|c|c|}
\hline Date of & change & New rate & Date of change & New rate & Date of change & New rate \\
\hline 198 & 6 Jan 9 & 12.50 & Oct 5 & 15.00 & & \\
\hline & Mar 19 & 11.50 & & & 1999 Jan 7 & 6.00 \\
\hline & Apr 8 & $11.00-11.50$ & 1990 Oct 8 & 14.00 & Feb 4 & 5.50 \\
\hline & Apr 21 & 10.50 & 1991 Feb 13 & 13.50 & Jun 10 & 5.00 \\
\hline & May 23 & $10.00-10.50$ & Feb 27 & 13.00 & Sep 8 & $5.00-5.25$ \\
\hline & May 27 & 10.00 & Mar 22 & 12.50 & Sep 10 & 5.25 \\
\hline & Oct 14 & $10.00-11.00$ & Apr 12 & 12.00 & Nov 4 & 5.50 \\
\hline \multirow{2}{*}{\multicolumn{2}{|c|}{ Oct 15}} & 11.00 & May 24 & 11.50 & & \\
\hline & & & Jul 12 & 11.00 & 2000 Jan 13 & 5.75 \\
\hline \multirow{12}{*}{1987} & Mar 10 & 10.50 & Sep 4 & 10.50 & Feb 10 & 6.00 \\
\hline & Mar 18 & $10.00-10.50$ & & & & \\
\hline & Mar 19 & 10.00 & 1992 May 5 & 10.00 & 2001 Feb 8 & 5.75 \\
\hline & Apr 28 & $9.50-10.00$ & Sep $16^{2}$ & 12.00 & Apr 5 & 5.50 \\
\hline & Apr 29 & 9.50 & Sep $17^{2}$ & $10.00-12.00$ & May 10 & 5.25 \\
\hline & May 11 & 9.00 & Sep 18 & 10.00 & Aug 2 & 5.00 \\
\hline & Aug 6 & $9.00-10.00$ & Sep 22 & 9.00 & Sep 18 & 4.75 \\
\hline & Aug 7 & 10.00 & Oct 16 & $8.00-9.00$ & Oct 4 & 4.50 \\
\hline & Oct 23 & $9.50-10.00$ & Oct 19 & 8.00 & Nov 8 & 4.00 \\
\hline & Nov 4 & $9.00-9.50$ & & & 2003 Feb 6 & 3.75 \\
\hline & Nov 5 & 9.00 & 1993 Jan 26 & 6.00 & Jul 10 & 3.50 \\
\hline & Dec 4 & 8.50 & Nov 23 & 5.50 & Nov 6 & 3.75 \\
\hline & 9.00 & 1994 Feb 8 & 5.25 & 2004 Feb 5 & 4.00 \\
\hline \multicolumn{2}{|c|}{$\begin{array}{r}1988 \text { Feb } 2 \\
\text { Mar } 17\end{array}$} & $8.50-9.00$ & Sep 12 & 5.75 & May 6 & 4.25 \\
\hline \multirow{2}{*}{\multicolumn{2}{|c|}{$\begin{array}{l}\text { Mar } 18 \\
\text { Apr } 11\end{array}$}} & 8.50 & Dec 7 & 6.25 & Jun 10 & 4.50 \\
\hline & & 8.00 & & & Aug 5 & 4.75 \\
\hline \multicolumn{2}{|r|}{ May 17} & $7.50-8.00$ & 1995 Feb $2^{2}$ & $6.25-6.75$ & & \\
\hline \multicolumn{2}{|r|}{ May 18} & 7.50 & Feb 3 & 6.75 & 2005 Aug 4 & 4.50 \\
\hline \multirow{2}{*}{\multicolumn{2}{|c|}{$\begin{array}{l}\text { Jun } 2 \\
\text { Jun } 3\end{array}$}} & $7.50-8.00$ & Dec 13 & 6.50 & & \\
\hline & & 8.00 & & & 2006 Aug 3 & 4.75 \\
\hline \multicolumn{2}{|r|}{ Jun 6} & $8.00-8.50$ & 1996 Jan 18 & 6.25 & Nov 9 & 5.00 \\
\hline \multicolumn{2}{|r|}{ Jun 7} & 8.50 & Mar 8 & 6.00 & & \\
\hline \multicolumn{2}{|r|}{ Jun 22} & $8.50-9.00$ & Jun 6 & 5.75 & 2007 Jan 11 & 5.25 \\
\hline \multicolumn{2}{|r|}{ Jun 23} & 9.00 & Oct 30 & $5.75-6.00$ & May 10 & 5.50 \\
\hline \multicolumn{2}{|r|}{ Jun 28} & $9.00-9.50$ & Oct 31 & 6.00 & Jul 5 & 5.75 \\
\hline \multicolumn{2}{|r|}{ Jun 29} & 9.50 & & & $\operatorname{Dec} 6$ & 5.50 \\
\hline \multicolumn{2}{|r|}{ Jul 4} & $9.50-10.00$ & 1997 May 6 & 6.25 & & \\
\hline \multicolumn{2}{|r|}{$\begin{array}{r}\text { Jul } 5 \\
\text { Jul } 18\end{array}$} & 10.00 & Jun 6 & $6.25-6.50$ & 2008 Feb 7 & 5.25 \\
\hline \multicolumn{2}{|r|}{ Aug 8} & $10.50-11.00$ & Aug 7 & 7.00 & Nov 6 & 3.00 \\
\hline \multirow{2}{*}{\multicolumn{2}{|c|}{$\begin{array}{r}\text { Aug 9 } \\
\text { Aug } 25\end{array}$}} & 11.00 & Nov 6 & 7.25 & $\operatorname{Dec} 4$ & 2.00 \\
\hline & & $11.00-12.00$ & & & & \\
\hline \multicolumn{2}{|r|}{ Aug 26} & 12.00 & 1998 Jun 4 & 7.50 & 2009 Jan 8 & 1.50 \\
\hline \multirow{2}{*}{\multicolumn{2}{|c|}{ Nov 25}} & 13.00 & Oct 8 & 7.25 & Feb 5 & 1.00 \\
\hline & & & Nov 5 & 6.75 & Mar 5 & 0.50 \\
\hline 1989 & May 24 & 14.00 & Dec 10 & 6.25 & & \\
\hline
\end{tabular}

1 Data obtained from Barclays Bank, Lloyds/TSB Bank, HSBC Bank and

National Westminster Bank whose rates are used to compile this series.

Source: Bank of England: 02076013644

2 Where all the rates did not change on the same day a spread is shown. 


\subsection{Average three month sterling money market rates ${ }^{1}$}

Percentage rates

2004

2005

2006

2008

Treasury bills: ${ }^{2}$ KDMM

January

February

March

April

June

July

August

September

October

November

December

$\begin{array}{llll}6.80 & 5.28 & 5.72 & 5.49 \\ 6.88 & 5.04 & 5.83 & 5.46 \\ 6.95 & 4.92 & 5.86 & 5.23 \\ 7.00 & 4.90 & 5.92 & 5.12 \\ 7.01 & 4.93 & 5.95 & 4.98 \\ 7.29 & 4.76 & 5.85 & 4.99 \\ & & & \\ 7.22 & 4.76 & 5.83 & 5.01 \\ 7.19 & 4.85 & 5.81 & 4.72 \\ 6.94 & 5.12 & 5.78 & 4.43 \\ 6.54 & 5.23 & 5.75 & 4.16 \\ 6.31 & 5.20 & 5.68 & 3.78 \\ 5.72 & 5.46 & 5.62 & 3.83\end{array}$

3.83
3.87
3.97
3.97
3.95
3.98

3.84
3.77
3.79
3.75
3.80
3.84

3.80
3.50
3.47
3.45
3.44
3.47

3.31
3.40
3.52
3.65
3.81
3.83

3.92
4.01
4.13
4.20
4.40
4.61

4.67
4.71
4.69
4.68
4.66
4.68

4.66
4.69
4.77
4.70
4.66
4.62
4.46
4.41
4.40
4.40
4.42
4.43

4.39

4.38

4.40

4.42

4.50

4.54

4.53

4.75

4.84

4.94

5.01
5.08

2007

Eligible bill: $\mathrm{KDMY}^{3}$

January

February

March

April

May

June

$7.28 \quad 5.63$

$5.63 \quad 5.90$

5.64

3.91

3.87

3.94

4.75

$3.99 \quad 3.54$

$4.04 \quad 3.52$

$4.01 \quad 3.52$

$4.19 \quad 4.88$

$4.28 \quad 4.84$

$\begin{array}{ll}4.28 & 4.84 \\ 4.42 & 4.80\end{array}$

July

August

September

October

November

7.24
7.20
7.42

5.08
4.94

$6.05 \quad 5.21$

$\begin{array}{ll}6.09 & 5.06 \\ 6.03 & 5.08\end{array}$

4.04

3.45

4.68

4.76

$7.49 \quad 4.89$

7.40

7.20

6.91

$4.89 \quad 5.97$

$\begin{array}{ll}4.94 & 5.97 \\ 5.16 & 5.95\end{array}$

5.07

3.94

3.39

3.86
3.86

$\begin{array}{ll}3.86 & 3.59 \\ 3.82 & 3.69\end{array}$

$5.92 \quad 4.26$

$5.88 \quad 3.85$

3.84

4.75

4.85

December

6.52
6.05

5.59

5.78

3.88
3.90

4.79

4.78

4.77

Interbank rate: AMIJ

January

February

March

April

May

June

July

August

September

Octobe

November

December

$\begin{array}{ll}7.48 & 5.80 \\ 7.46 & 5.43 \\ 7.48 & 5.30 \\ 7.44 & 5.23 \\ 7.41 & 5.25 \\ 7.63 & 5.12 \\ & \\ 7.71 & 5.07 \\ 7.66 & 5.18 \\ 7.38 & 5.32 \\ 7.14 & 5.94 \\ 6.89 & 5.78 \\ 6.38 & 5.97\end{array}$

$\begin{array}{ll}6.06 & 5.76 \\ 6.15 & 5.69 \\ 6.15 & 5.47 \\ 6.21 & 5.33 \\ 6.23 & 5.17 \\ 6.14 & 5.19 \\ & \\ 6.11 & 5.19 \\ 6.14 & 4.93 \\ 6.12 & 4.65 \\ 6.08 & 4.36 \\ 6.00 & 3.93 \\ 5.89 & 3.99\end{array}$

3.98
3.98
4.06
4.11
4.08
4.11

3.99
3.92
3.93
3.90
3.91
3.95

3.91
3.69
3.58
3.58
3.57
3.57

3.42
3.45
3.63
3.73
3.91
3.95

$\begin{array}{ll}3.99 & 4.80 \\ 4.10 & 4.82 \\ 4.23 & 4.92 \\ 4.33 & 4.88 \\ 4.46 & 4.83 \\ 4.73 & 4.78 \\ & \\ 4.79 & 4.59 \\ 4.89 & 4.53 \\ 4.87 & 4.54 \\ 4.83 & 4.53 \\ 4.82 & 4.56 \\ 4.81 & 4.59\end{array}$

Certificate of deposits: KOSA

January
February
March
April
May
June
July
August
September
October
November
December

7.44
7.42

7.42

7.43

$7.40-5.19$

$\begin{array}{ll}7.37 & 5.22 \\ 7.59 & 5.09\end{array}$

$\begin{array}{ll}7.59 & 5.09\end{array}$

$7.66 \quad 5.03$

$\begin{array}{ll}7.66 & 5.03 \\ 7.61 & 5.14\end{array}$

$7.34 \quad 5.28$

$7.09 \quad 5.86$

$6.82-5.72$

$\begin{array}{ll}6.32 & 5.89\end{array}$

Local authority deposits: $\mathrm{KDPX}^{4}$

January

February

March

April

May

July

August

September

October

Novembe

December

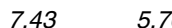

$\begin{array}{ll}7.43 & 5.76 \\ 7.40 & 5.38 \\ 7.40 & 5.27\end{array}$

7.40

7.34
7.56

7.64
7.55
7.35

7.35

7.08

6.85
6.35
6.02

$6.10-5.73$

6.17

6.19

6.10

6.08

6.09

6.08

6.05

$\begin{array}{ll}5.98 & 3.91 \\ 5.85 & 3.96\end{array}$

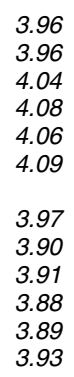

3.90

$$
3.68
$$$$
3.57
$$

3.57

3.56

3.56

3.41

3.44

3.62

3.72

3.90

3.94
3.98

4.09

4.22

4.32

4.45
4.72

4.79

4.89

4.87

4.83

4.81

4.80
4.80
4.82
4.91
4.86
4.82
4.78
4.60
4.53
4.54
4.52
4.56
4.58

4.54

4.52

4.53

4.57

4.65
4.69

4.68

4.89

4.98

5.09

5.18

$\begin{array}{ll}5.45 & 5.61 \\ 5.51 & 5.60 \\ 5.52 & 5.85 \\ 5.69 & 5.89 \\ 5.84 & 5.79 \\ 5.94 & 5.89 \\ & \\ 6.11 & 5.80 \\ 6.35 & 5.75 \\ 6.54 & 5.86 \\ 6.21 & 6.16 \\ 6.34 & 4.40 \\ 6.35 & 3.21\end{array}$

1 A full definition of these series is given in Section 7 of the ONS Financial 3 This series discontinued at end of August 2005

Statistics Explanatory Handbook.

2 Average rate of discount at weekly (Friday) tender.

4 This series discontinued at end of December 2004

$\begin{array}{llllll}5.76 & 6.03 & 5.73 & 3.85 & 3.87 & 3.91 \\ 5.38 & 6.09 & 5.62 & 3.88 & 3.61 & 4.08 \\ 5.27 & 6.08 & 5.39 & 4.01 & 3.55 & 4.12 \\ 5.17 & 6.12 & 5.26 & 4.05 & 3.54 & 4.31 \\ 5.19 & 6.14 & 5.13 & 4.06 & 3.54 & 4.45 \\ 5.07 & 6.09 & 5.10 & 4.05 & 3.57 & 4.75 \\ & & & & & \\ 5.01 & 6.04 & 5.12 & 3.95 & 3.39 & 4.82 \\ 5.11 & 6.06 & 4.86 & 3.87 & 3.43 & 4.92 \\ 5.19 & 6.05 & 4.58 & 3.88 & 3.61 & 4.90 \\ 5.83 & 6.03 & 4.29 & 3.86 & 3.71 & 4.85 \\ 5.64 & 5.96 & 3.82 & 3.87 & 3.90 & 4.84 \\ 5.88 & 5.80 & 3.87 & 3.93 & 3.92 & 4.82\end{array}$

3.91

4.12

4.75

4.92

4.90

4.85 
Sterling exchange

rate index $(1990=100)^{2}$ AGBG

January

February

March

April

May

June

July

August

September

October

November

December

Effective Sterling exchang

rate index (Jan $2005=100)$ BK67

January

February

March

April

May

June

July

August

September

October

November

December

Sterling/US Dollar AUSS

January

February

March

April

May

June

July

August

September

October

November

December

Sterling/Euro THAP

January

February

March

April

May

June

July

August

September

October

November

December

$1999 \quad 2000$

2001

2002

2003

2004

2005

2006

2007

2008

$\begin{array}{rrrrrrrrr}104.7 & 99.6 & 108.5 & 104.4 & 106.9 & 104.0 & 102.4 & 102.1 & 102.7 \\ 104.7 & 100.8 & 108.4 & 104.1 & 107.4 & 102.4 & 104.8 & 103.3 & 102.8 \\ 106.8 & 102.8 & 108.4 & 105.0 & 106.5 & 100.6 & 105.0 & 103.2 & 102.1 \\ 107.1 & 103.4 & 110.1 & 105.8 & 107.1 & 99.8 & 105.2 & 104.4 & 101.9 \\ 103.4 & 104.2 & 108.5 & 106.6 & 105.3 & 97.9 & 104.6 & 103.6 & 104.1 \\ 105.4 & 104.7 & 104.6 & 106.8 & 103.6 & 99.6 & 105.8 & 104.9 & . . \\ & & & & & & & & \\ 105.3 & 103.5 & 105.6 & 107.2 & 105.3 & 99.4 & 105.9 & 102.1 & . . \\ 104.6 & 103.3 & 107.4 & 105.1 & 105.4 & 99.0 & 105.2 & 102.8 & . . \\ 103.3 & 104.7 & 106.2 & 106.1 & 106.5 & 99.2 & 103.3 & 103.9 & . . \\ 100.7 & 105.4 & 109.2 & 105.8 & 106.7 & 99.8 & 102.2 & 103.1 & . . \\ 100.6 & 105.7 & 107.3 & 106.1 & 105.9 & 100.4 & 101.7 & 103.2 & . . \\ 100.4 & 106.7 & 106.4 & 106.5 & 105.5 & 100.3 & 103.2 & 103.3 & . .\end{array}$

$\begin{array}{rrrrrrrrrrr}100.4 & 96.4 & 102.9 & 98.4 & 100.5 & 99.9 & 100.1 & 100.0 & 99.1 & 105.4 & 96.4 \\ 100.2 & 97.2 & 102.5 & 98.0 & 100.7 & 98.4 & 102.5 & 101.0 & 98.9 & 104.9 & 95.9 \\ 101.9 & 98.8 & 102.3 & 98.7 & 100.0 & 96.7 & 102.2 & 101.1 & 98.4 & 103.4 & 94.6 \\ 102.1 & 98.9 & 103.6 & 99.3 & 100.7 & 96.0 & 102.1 & 102.0 & 98.3 & 104.1 & 92.8 \\ 99.0 & 99.6 & 101.7 & 99.6 & 99.3 & 94.8 & 101.8 & 101.0 & 101.3 & 103.7 & 92.8 \\ 101.0 & 99.6 & 98.6 & 99.5 & 98.2 & 96.6 & 103.0 & 101.8 & 100.8 & 104.3 & 93.0 \\ & & & & & & & & & & \\ 100.9 & 98.4 & 99.4 & 100.0 & 100.3 & 96.0 & 103.1 & 98.8 & 100.8 & 105.0 & 93.2 \\ 100.3 & 98.7 & 100.6 & 98.7 & 100.3 & 95.4 & 102.4 & 99.7 & 102.8 & 104.4 & 91.6 \\ 99.8 & 99.9 & 99.1 & 99.8 & 101.4 & 95.7 & 100.7 & 100.7 & 102.9 & 103.1 & 89.9 \\ 97.6 & 100.7 & 101.7 & 99.6 & 101.6 & 96.8 & 99.8 & 99.7 & 103.0 & 102.6 & 89.3 \\ 97.3 & 100.6 & 100.0 & 99.7 & 101.0 & 97.3 & 99.7 & 99.4 & 103.3 & 101.7 & 83.4 \\ 97.3 & 101.2 & 99.8 & 100.2 & 100.8 & 97.8 & 101.3 & 99.6 & 104.3 & 99.8 & 78.1\end{array}$

1 Working day average. A full definition of these series is given in Section 7 of

the ONS Explanatory Handbook.

$\begin{array}{lllllllllll}1.6 & 1.7 & 1.6 & 1.5 & 1.4 & 1.6 & 1.8 & 1.9 & 1.8 & 2.0 & 2.0 \\ 1.6 & 1.6 & 1.6 & 1.5 & 1.4 & 1.6 & 1.9 & 1.9 & 1.7 & 2.0 & 2.0 \\ 1.7 & 1.6 & 1.6 & 1.4 & 1.4 & 1.6 & 1.8 & 1.9 & 1.7 & 1.9 & 2.0 \\ 1.7 & 1.6 & 1.6 & 1.4 & 1.4 & 1.6 & 1.8 & 1.9 & 1.8 & 2.0 & 2.0 \\ 1.6 & 1.6 & 1.5 & 1.4 & 1.5 & 1.6 & 1.8 & 1.9 & 1.9 & 2.0 & 2.0 \\ 1.7 & 1.6 & 1.5 & 1.4 & 1.5 & 1.7 & 1.8 & 1.8 & 1.8 & 2.0 & 2.0 \\ & & & & & & & & & & \\ 1.6 & 1.6 & 1.5 & 1.4 & 1.6 & 1.6 & 1.8 & 1.8 & 1.8 & 2.0 & 2.0 \\ 1.6 & 1.6 & 1.5 & 1.4 & 1.5 & 1.6 & 1.8 & 1.8 & 1.9 & 2.0 & 1.9 \\ 1.7 & 1.6 & 1.4 & 1.5 & 1.6 & 1.6 & 1.8 & 1.8 & 1.9 & 2.0 & 1.8 \\ 1.7 & 1.7 & 1.5 & 1.5 & 1.6 & 1.7 & 1.8 & 1.8 & 1.9 & 2.0 & 1.7 \\ 1.7 & 1.6 & 1.4 & 1.4 & 1.6 & 1.7 & 1.9 & 1.7 & 1.9 & 2.1 & 1.5 \\ 1.7 & 1.6 & 1.5 & 1.4 & 1.6 & 1.8 & 1.9 & 1.7 & 2.0 & 2.0 & 1.5\end{array}$

2 Series discontinued from 31 May 2006. 
$1997 \quad 1998$ 1999 2000 2001 2002 2003 2004 2005 $2006 \quad 2007$

Nominal Five Year Yield ZBRG
January

February

March

April

June

July

August

September

October

November

December

Nominal Ten Year Yield ZBRH

January

February

March

April

May

June

July

August

October

November

December

Nominal Twenty Year Yield ZBRI

January

February

March

April

May

June

July

August

September

October

November

Real Ten Year Yield ZBRJ

January

February

March

April

May

June

July

August

September

November

December

Real Twenty Year Yield ZBRK

January

February

March

April

May

June

July

August

September

October

November

December
December

$\begin{array}{ll}7.15 & 6.18 \\ 6.82 & 6.1 \\ 7.07 & 6.09 \\ 7.28 & 5.93 \\ 6.94 & 5.95 \\ 6.96 & 6.04 \\ 7.01 & 6.1 \\ 6.97 & 5.80 \\ 6.72 & 5.32 \\ 6.51 & 4.94 \\ 6.69 & 4.9 \\ 6.46 & 4.51\end{array}$

\section{$6.18 \quad 4.30$}

6.10

6.10
6.09
5.93
5.95

5.95
6.04

4.46
4.69

4.69
4.66

4.95

5.28

6.12
5.80

5.32

4.94

4.92
4.51

5.49

5.75
6.00

6.00

6.25
5.86

5.90

\subsection{8}

6.13

5.89

5.80

5.82
5.61

5.58

5.65

5.65

5.46

5.33
5.14

5.07
5.04
4.86
4.96
5.14
5.25

5.26
5.03
4.90
4.74
4.55
4.88

4.90

4.94

5.22

5.21

5.22
5.05

4.88

4.54

4.31

4.36

4.38

4.34

$\begin{array}{ll}4.15 & 4.61 \\ 3.85 & 4.63 \\ 3.93 & 4.56 \\ 4.09 & 4.80 \\ 3.85 & 5.01 \\ 3.72 & 5.15 \\ & \\ 3.98 & 5.07 \\ 4.36 & 4.96 \\ 4.46 & 4.83 \\ 4.73 & 4.65 \\ 4.91 & 4.58 \\ 4.71 & 4.43\end{array}$

$\begin{array}{lll}4.43 & 4.11 & 5.06 \\ 4.53 & 4.17 & 5.08 \\ 4.73 & 4.33 & 5.00 \\ 4.54 & 4.48 & 5.20 \\ 4.31 & 4.67 & 5.32 \\ 4.17 & 4.69 & 5.59 \\ & & \\ 4.16 & 4.69 & 5.55 \\ 4.23 & 4.74 & 5.25 \\ 4.12 & 4.67 & 5.02 \\ 4.26 & 4.76 & 4.95 \\ 4.29 & 4.73 & 4.64 \\ 4.21 & 4.80 & 4.57\end{array}$

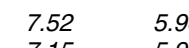

7.15

7.41

7.58

7.08
7.04

6.92
6.97
6.70

6.97
6.70
6.37

6.70
6.37
6.46

6.46

6.22
$5.96 \quad 4.24$

5.91

5.85

5.69

5.73
5.60

\subsection{5}

5.65
5.41

5.03
4.93

4.93
4.83

4.83
4.44
$4.24 \quad 5.62$

$4.39 \quad 5.44$

$4.60 \quad 5.18$

$4.53 \quad 5.14$

$\begin{array}{ll}4.83 & 5.23 \\ 5.07 & 5.05\end{array}$

$5.24 \quad 5.09$

$\begin{array}{ll}5.24 & 5.09 \\ 5.25 & 5.18\end{array}$

$5.51 \quad 5.25$

$5.68 \quad 5.09$

$\begin{array}{ll}5.11 & 4.98 \\ 5.19 & 4.80\end{array}$

4.75
4.90
4.64
4.90
5.05
5.11

5.10
4.88
4.91
4.77
4.58
4.83

4.85

4.90

4.90
5.18
5.19

5.22
5.05

\subsection{5}

4.68

4.47
4.60

4.60
4.62

4.55

4.76
4.78
4.67
4.92
5.06
5.13

5.04
4.95
4.86
4.72
4.65
4.49

4.50

4.50
4.54
4.74

4.74

4.58

4.38
4.25

4.28

4.29

4.17

4.31

4.26

4.02

4.10

4.26

$\begin{array}{ll}4.58 & 5.03 \\ 4.60 & 5.31\end{array}$

$4.59 \quad 5.29$

$4.58 \quad 5.05$

$4.47 \quad 4.91$

$4.53 \quad 4.88$

$\begin{array}{ll}4.45 & 4.67 \\ 4.53 & 4.65\end{array}$

\subsection{4}

$\begin{array}{lll}5.94 & 4.36 & 4.45\end{array}$

$5.88 \quad 4.44$

5.78
5.61

$5.61 \quad 4.53$

$5.67 \quad 4.75$

7.16

7.08

5.42

6.80

6.86

6.64

6.36

6.37

$5.45 \quad 4.67$

$5.30 \quad 4.53$

4.91

4.87

4.56
4.07

4.47

4.20
4.45
4.38

4.38
4.25

4.35

4.40

4.37

4.33

4.33
4.42

4.42
4.45

4.76

4.87

4.69

4.72

4.99

5.02

5.08

4.93

4.90

4.69

4.82

4.57

4.40

4.54

4.60

4.59
4.69

4.69
4.72

4.61

4.79

4.89

4.80
4.69
4.65

4.65

4.59

4.50

4.42
4.45

4.45
4.45

4.65

4.53

4.36

4.23

4.24

4.27

4.16

4.30

4.28
4.19

$\begin{array}{ll}4.05 & 4.87 \\ 4.13 & 4.90 \\ 4.29 & 4.82 \\ 4.48 & 5.04 \\ 4.62 & 5.14 \\ 4.64 & 5.42 \\ & \\ 4.63 & 5.40 \\ 4.64 & 5.13 \\ 4.54 & 4.96 \\ 4.61 & 4.92 \\ 4.55 & 4.68 \\ 4.62 & 4.64\end{array}$

3.45

3.27

$$
3.43
$$

$$
3.56
$$

3.56
3.57
3.66

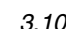

$3.10 \quad 2.00$

3.06

3.00

2.91

2.92

2.85

1.91
1.85

1.70

1.91
1.89

1.89 


\subsection{Average rates on representative British Government Stocks ${ }^{1}$}

Percentage rates

1998

1999

2000

2001

2002

2003

2004

2005

2006

2007

5 Year Conventional Rate ${ }^{2}$ KORP

January

February

March

April

May

June

July

August

September

October

November

December

$\begin{array}{lll}6.33 & 4.25 & 6.36 \\ 6.24 & 4.41 & 6.23 \\ 6.26 & 4.65 & 6.01 \\ 6.11 & 4.66 & 5.95 \\ 6.14 & 4.93 & 5.97 \\ 6.31 & 5.27 & 5.78 \\ & & \\ 6.14 & 5.49 & 5.75 \\ 5.84 & 5.80 & 5.81 \\ 5.34 & 6.04 & 5.81 \\ 4.88 & 6.24 & 5.66 \\ 4.86 & 5.89 & 5.50 \\ 4.45 & 5.91 & 5.27\end{array}$

5.17
5.13
4.94
4.97
5.15
5.32

5.34
5.09
4.94
4.78
4.59
4.88

4.94
4.96
5.23
5.26
5.48
5.10

4.92
4.57
4.25
4.38
4.40
4.34

4.15

3.88

3.93

4.08
3.83

3.83

3.72

4.30

4.42

4.70

4.88

4.68

$\begin{array}{llr}4.59 & 4.43 & 4.27 \\ 4.46 & 4.61 & 4.31 \\ 4.44 & 4.77 & 4.41 \\ 4.66 & 4.58 & 4.44 \\ 4.89 & 4.36 & 4.44 \\ 5.08 & 4.24 & 4.66 \\ & & \\ 4.98 & 4.11 & 4.62 \\ 4.88 & 4.22 & 4.84 \\ 4.76 & 4.18 & 4.91 \\ 4.57 & 4.23 & 5.00 \\ 4.52 & 4.31 & 4.39 \\ 4.42 & 4.27 & -\end{array}$

10 year Conventional Rate KORQ

January

February

March

April

May

June

July

August

September

Octobe

November

December

20 Year Conventional Rate KORR

$\begin{array}{ll}6.07 & 4.16 \\ 6.02 & 4.32 \\ 5.97 & 4.5 \\ 5.81 & 4.48 \\ 5.85 & 4.77 \\ 5.77 & 5.02 \\ & \\ 5.67 & 5.20 \\ 5.56 & 5.24 \\ 5.10 & 5.5 \\ 4.93 & 5.70 \\ 4.87 & 5.16 \\ 4.49 & 5.24\end{array}$

$\begin{array}{llll}4.16 & 5.75 & 4.86 & 4.84 \\ 4.32 & 5.56 & 4.88 & 4.91 \\ 4.54 & 5.29 & 4.75 & 5.15 \\ 4.48 & 5.25 & 4.95 & 5.23 \\ 4.77 & 5.35 & 5.13 & 5.51 \\ 5.02 & 5.15 & 5.09 & 5.06 \\ & & & \\ 5.20 & 5.18 & 5.16 & 4.94 \\ 5.24 & 5.27 & 4.92 & 4.66 \\ 5.52 & 5.32 & 4.92 & 4.46 \\ 5.70 & 5.15 & 4.76 & 4.57 \\ 5.16 & 5.06 & 4.58 & 4.59 \\ 5.24 & 4.88 & 4.88 & 4.52\end{array}$

$$
\begin{aligned}
& 4.37 \\
& 4.25 \\
& 4.51 \\
& 4.64 \\
& 4.26 \\
& 4.38 \\
& \\
& 4.23 \\
& 4.59 \\
& 4.69 \\
& 4.89 \\
& 5.04 \\
& 4.94
\end{aligned}
$$

4.78

4.75

4.65

4.91

5.07
5.19

4.51
4.60

4.79

4.60

4.38
4.23

5.10

4.99

4.89

4.73

4.66

4.20

4.25

4.16
4.31

4.33

4.27

$\begin{array}{lll}4.19 & 5.21 & 4.33 \\ 4.25 & 5.25 & 4.28 \\ 4.39 & 5.15 & 4.00 \\ 4.47 & 5.34 & 4.28 \\ 4.39 & 5.39 & 4.75 \\ 4.74 & 5.74 & 5.15 \\ & & \\ 4.73 & 5.70 & 5.00 \\ 4.78 & 5.39 & 4.63 \\ 4.74 & 5.13 & 4.23 \\ 4.85 & 5.07 & 3.76 \\ 4.84 & 4.70 & 2.86 \\ 4.93 & 4.60 & 2.26\end{array}$

January

February

March

April

May

June

July

August

September

October

November

December

10 Year Index-Linked Rate KORS

6.04

5.98

5.90

5.73

5.79

5.59

5.63

5.43

5.02

4.92

4.79

4.49

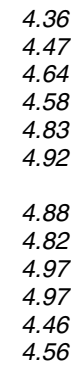

4.91

4.80

4.64

4.71

4.77

4.68

4.70

4.79

4.90

4.84

4.64

4.51

\subsection{2}

4.58

4.56

4.84

4.98

5.10

5.05

4.83
4.94

4.94

4.80
4.55

4.75

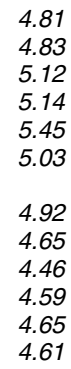

4.46

4.37

4.51

4.64

4.44
4.38

4.59

4.67

4.74

4.85

4.93

4.80

4.73

4.80

4.69

4.91

5.03
5.07

5.07

4.99

4.88

4.83

4.73

4.64
4.53

4.55

4.55
4.58

4.79

4.63

4.43

4.30

4.33
4.34

4.34
4.24

4.37

4.31

4.22

\subsection{5}

4.13

4.28

4.38

4.38
4.59
4.60

4.60

4.58

4.49

4.53

4.48
4.41

$\begin{array}{ll}4.77 & 4.54 \\ 4.80 & 4.68 \\ 4.73 & 4.54 \\ 4.95 & 4.77 \\ 5.08 & 4.95 \\ 5.32 & 5.22 \\ & \\ 5.28 & 5.09 \\ 5.04 & 4.62 \\ 4.89 & 4.67 \\ 4.92 & 4.65 \\ 4.73 & 4.33 \\ 4.71 & 3.70\end{array}$

January

February

March

April

May

June

July

August

October

November

December

2.11

2.21

2.61

2.07

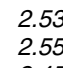

2.55

2.45

2.58
2.35

1.81
1.88

1.88

1.90

1.74
1.59

1.67

2.46

2.37
2.24

2.42

2.39

1.89

1.99

2.08

2.16
1.97

1.88
1.90
1.76
1.94
2.10
2.17
2.1
2.04
1.95
1.83
1.85
1.74

1.73

1.81

1.99

1.83

1.71
1.67

1.48

1.54
1.65

1.65

1.96
1.94

1.66

1.63

1.49

1.89

1.64

\subsection{2}

1.80

1.76

1.89

1.87
1.96

$\begin{array}{ll}2.24 & 1.60 \\ 2.27 & 1.29\end{array}$

$2.18 \quad 0.76$

$2.61 \quad 1.12$

$2.59 \quad 1.48$

$2.68 \quad 1.61$

$2.37 \quad 1.64$

$2.01 \quad 1.69$

$\begin{array}{ll}1.99 & 2.38 \\ 1.70 & 3.44\end{array}$

$\begin{array}{ll}1.70 & 3.44 \\ 1.80 & 3.59\end{array}$

20 Year Index-Linked rate KORT

January

February

March

April

May

June

July

August

September

October

November

December

$\begin{array}{ll}3.01 & 2.06 \\ 3.01 & 1.97 \\ 2.92 & 1.93 \\ 2.80 & 1.81 \\ 2.79 & 1.99 \\ 2.61 & 1.97 \\ & \\ 2.56 & 1.97 \\ 2.51 & 2.12 \\ 2.51 & 2.23 \\ 2.58 & 2.18 \\ 2.35 & 1.91 \\ 2.12 & 1.88\end{array}$

$\begin{array}{lll}2.01 & 1.96 & 2.35 \\ 1.98 & 1.99 & 2.36 \\ 1.83 & 2.09 & 2.39 \\ 1.90 & 2.35 & 2.32 \\ 1.97 & 2.41 & 2.43 \\ 1.94 & 2.38 & 2.23 \\ & & \\ 1.96 & 2.36 & 2.30 \\ 2.03 & 2.25 & 2.21 \\ 2.04 & 2.39 & 2.12 \\ 2.08 & 2.38 & 2.29 \\ 2.02 & 2.19 & 2.31 \\ 1.94 & 2.33 & 2.26\end{array}$

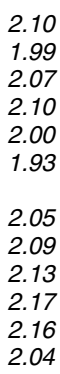

2.10

1.99
2.07

2.10

2.00

2.05

2.09
2.13

2.17

2.16
2.04

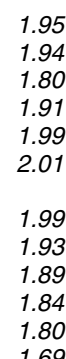

1.94
1.80
1.91
1.99
2.01

1.99
1.93
1.89
1.84
1.80
1.69

1.68

1.72

1.89

1.77

1.63

1.63

1.58

1.48

1.51

1.45
1.38

$\begin{array}{lll}1.17 & 1.63 & 1.25 \\ 1.22 & 1.65 & 1.30 \\ 1.34 & 1.57 & 1.04 \\ 1.38 & 1.77 & 1.24 \\ 1.56 & 1.91 & 1.38 \\ 1.61 & 2.07 & 1.34 \\ & & \\ 1.55 & 2.07 & 1.27 \\ 1.45 & 1.83 & 1.14 \\ 1.37 & 1.67 & 1.26 \\ 1.44 & 1.65 & 1.99 \\ 1.34 & 1.41 & 2.47 \\ 1.43 & 1.41 & 2.14 \\ & & \end{array}$




\begin{tabular}{|c|c|c|c|c|c|c|c|c|c|c|c|}
\hline & & $1998^{3}$ & $1999^{3}$ & $2000^{4}$ & 2001 & 2002 & 2003 & 2004 & 2005 & 2006 & 2007 \\
\hline \multicolumn{12}{|l|}{ Number and balance sheets } \\
\hline Societies on register (numbers) & KRNA & 71 & 69 & 67 & 65 & 65 & 63 & 63 & 63 & 60 & 59 \\
\hline Share investors (thousands) & KRNB & 21195 & 21774 & 22237 & 20311 & 20724 & 20897 & 20734 & 22090 & 22396 & 23038 \\
\hline Depositors (thousands) & KRNC & 909 & 722 & 740 & 568 & 511 & 520 & 525 & 449 & 472 & 460 \\
\hline Borrowers (thousands) & KRND & 3136 & 3044 & 3107 & 2750 & 2688 & 2679 & 2749 & 2822 & 2857 & 2941 \\
\hline
\end{tabular}

Assets and liabilities ( $£$ million)

Liabilities:
Shares
Deposits and wholesale
Taxation and other
General reserves
Other Capital
Assets:
Mortgages
Investments and cash
Other
Total

\begin{tabular}{|c|c|c|c|c|c|c|c|c|c|c|}
\hline KRNE & 103289.8 & 109137.6 & 119298.5 & 119815.2 & 132373.0 & 142456.9 & 153844.0 & 171935.0 & 188943.0 & 206782.5 \\
\hline KRNF & 33432.5 & 34579.2 & 43578.5 & 37985.2 & 37650.8 & 49204.4 & 63797.5 & 71703.7 & 82759.8 & 98364.5 \\
\hline KRNG & 2105.0 & 2259.8 & 2033.6 & 1532.0 & 1401.1 & 1498.8 & 1761.5 & 2983.6 & 4655.4 & 5417.8 \\
\hline KRNH & 8305.6 & 8733.0 & 9577.0 & 9152.2 & 9932.8 & 10592.8 & 11385.6 & 12151.1 & 12532.7 & 13607.5 \\
\hline KRNI & 1551.4 & 1529.9 & 1861.7 & 1391.7 & 1684.8 & 2510.3 & 3574.8 & 4534.9 & 5528.3 & 6099.8 \\
\hline RNK & 116284.5 & 120409.5 & 134100.1 & 128321.6 & 138884.0 & 156396.1 & 180172.4 & 203259.7 & 228095.9 & 257810 . \\
\hline KHVZ & 28343.0 & 31207.9 & 37900.8 & 37158.1 & 39201.8 & 44500.4 & 47810.8 & 51475.6 & 55102.9 & 60694.6 \\
\hline KRNN & 11386.7 & 5523.1 & 5746.2 & 5895.3 & 6367.0 & 6838.4 & 8163.1 & 10490.3 & 11220.4 & 11767.3 \\
\hline & 156014.2 & 157140.5 & 177747.1 & 171375.0 & 184452.8 & 207734.9 & 236146.3 & 265225.6 & 294419.2 & \\
\hline
\end{tabular}

Current transactions (£ million)

\begin{tabular}{llrrr} 
Mortgage advances & KRNU & 21988.3 & 23997.9 & 28233.6 \\
Management expenses & KRNX & 1501.7 & 1573.8 & 1640.7 \\
\hline 1 See chapter text. & \\
2 The figures for each year relate to accounting years ending on dates be- \\
tween 1 February of that year and 31 January of the following year. \\
3 The societies which have converted to the banking sector, namely Chel- \\
tenham \& Gloucester (August 1995), National \& Provincial (August 1996), \\
Alliance \& Leicester (April 1997), Halifax (June 1997), Woolwich (July \\
1997), Bristol \& West (July 1997), Northern Rock (October 1997), and \\
Birmingham Midshires (April 1999) have been included in flow figures (us- \\
ing flows up to the date of conversion), but have been excluded from the \\
end of year balances.
\end{tabular}
4 Bradford \& Bingley, which converted to the banking sector in December 2000,
is included within flow figures and the end of year balances.

Source: Financial Services Authority: 02070661000

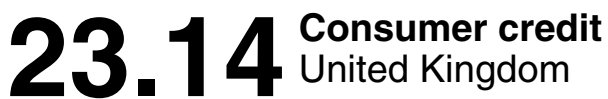

\begin{tabular}{|c|c|c|c|c|c|c|c|c|c|c|c|}
\hline & & 1998 & 1999 & 2000 & 2001 & 2002 & 2003 & 2004 & 2005 & 2006 & 2007 \\
\hline Total amount outstanding & VZRD & 106341 & 121547 & 135168 & 150802 & 169209 & 180649 & 198856 & 211038 & 212835 & 221687 \\
\hline Total net lending & VzQC & 15503 & 16133 & 15969 & 19673 & 23443 & 22401 & 25337 & 19666 & 13054 & 13471 \\
\hline \multicolumn{12}{|l|}{ of which } \\
\hline Credit cards & VZQS & 4858 & 5676 & 6686 & 6229 & 7579 & 8710 & 9998 & 6166 & 1951 & 2251 \\
\hline Other & VZQT & 10647 & 10457 & 9284 & 13445 & 15867 & 13692 & 15340 & 13499 & 11102 & 11221 \\
\hline Banks & AIKN & 11738 & 11057 & 13217 & 16055 & 17452 & 15269 & 19370 & 11317 & 9346 & 6075 \\
\hline Building societies' class 3 loans & ALPY & - & 12 & 112 & 63 & 180 & 177 & 172 & 238 & 217 & 260 \\
\hline Other consumer credit lenders & BM59 & 3764 & 5065 & 2640 & 3554 & 5811 & 6954 & 5796 & 8112 & 3489 & 7135 \\
\hline Total gross lending & VZQG & 134847 & 148623 & 160744 & 177452 & 196451 & 207255 & 221318 & 217467 & 207460 & 204631 \\
\hline
\end{tabular}

As from Dec 2006 the Bank of England has ceased to update the separate data on consumer credit provided by other specialist lenders, retailers and insurance companies previously contained in these tables. These categories have been merged into 'other consumer credit lenders'. 
Banking, insurance 23. 5 End-year assets and liabilities of investment trust companies, unit trusts ${ }^{1}$ and United Kingdom

$£$ million

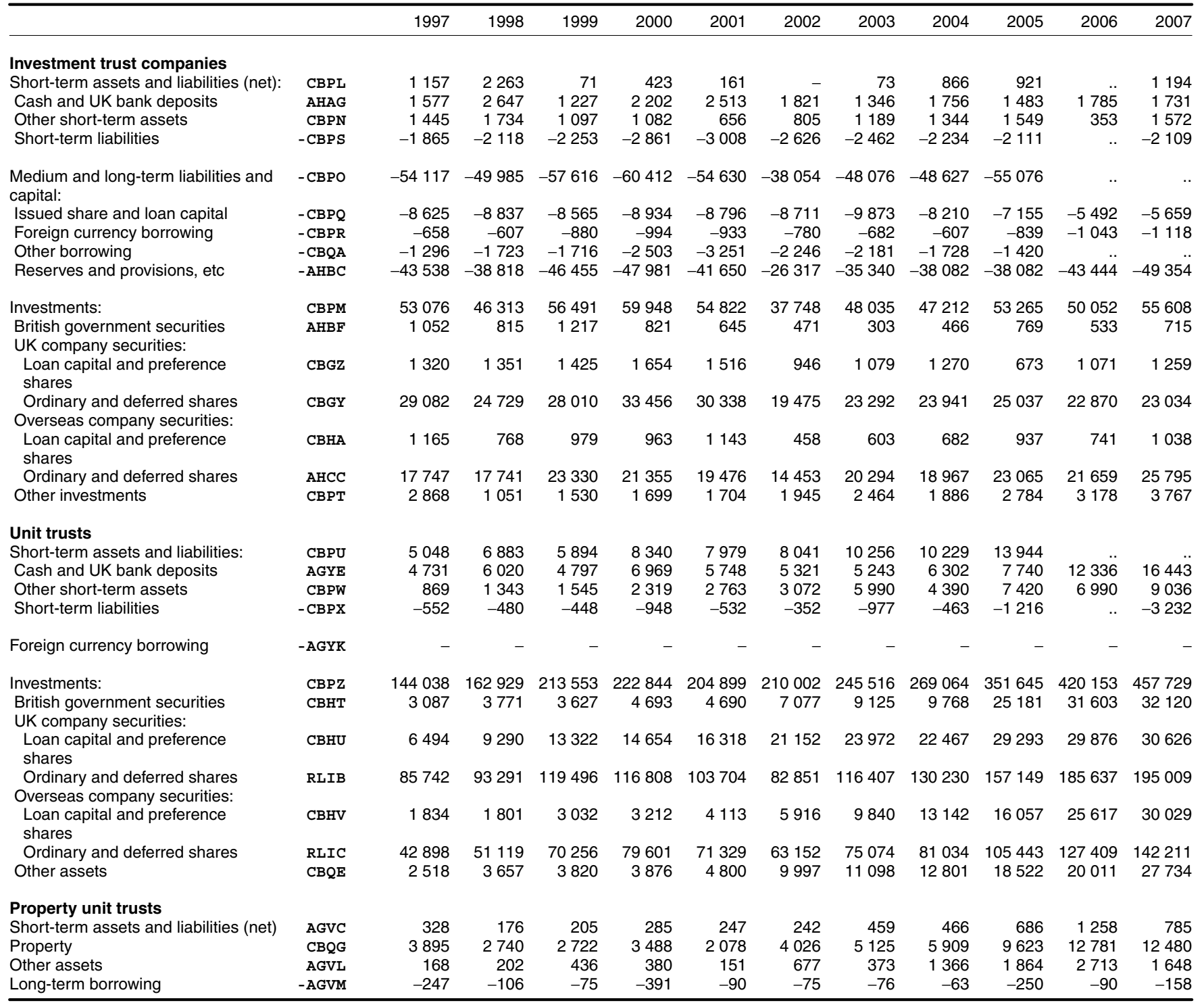

Note: Assets are shown as positive: liabilities as negative.

Source: Office for National Statistics: 01633812789

1 Including open ended investment companies (OEICs).

2 Investments are at market value.

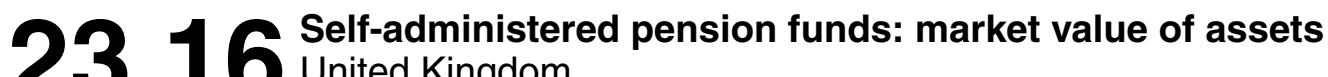 23.16 interes knosem End year}

£ million

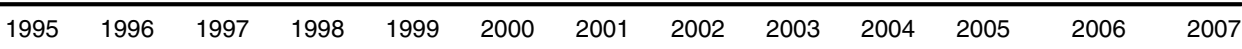

\begin{tabular}{|c|c|c|c|c|c|c|c|c|c|c|c|c|c|c|}
\hline \multicolumn{15}{|l|}{ Total pension funds ${ }^{1}$} \\
\hline Total net assets & AHVA & 508581 & 3879 & 656874 & 699191 & 812228 & 7651997 & 711572 & 6104416 & 6926947 & 761066 & 914955 & 010794 & 1023979 \\
\hline Short-term assets & RYIQ & 14 & 31521 & 35368 & 39005 & 32703 & 36638 & 31337 & 30700 & 46091 & 57476 & 73649 & 98691 & 99681 \\
\hline British government securities & AHVK & 52659 & 57783 & 80533 & 91084 & 98882 & 92458 & 83754 & 84461 & 88803 & 87579 & 94325 & 104910 & 113617 \\
\hline UK local authority long-term debt & AHVO & 83 & 89 & 156 & 183 & 133 & 177 & 125 & 42 & 8 & 4 & 4 & 2 & \\
\hline Ordinary shares & AHVP & 56625 & 276001 & 339687 & 334648 & 357230 & 299318 & 260696 & 1864371 & 1864261 & 180561 & 199 & 208473 & 152048 \\
\hline Other & AHVQ & 7064 & 6180 & & 8168 & 9258 & & 22301 & & & 43027 & & 54902 & 57541 \\
\hline \multicolumn{15}{|l|}{ Overseas company securities } \\
\hline Oth & AHVS & 184 & 4909 & 3851 & 3842 & 5099 & 12736 & 11781 & 11386 & 12475 & 15996 & 20502 & 31536 & 45470 \\
\hline UK loar & RLDQ & 34 & 83 & 160 & 22 & 14 & 7 & 3 & - & 35 & 44 & 6 & 6 & 12 \\
\hline rty and ground rent & AHWA & 21317 & 21637 & 24176 & 24355 & 31107 & 32945 & 30617 & & 30619 & 30552 & & 394 & 30304 \\
\hline Auth & AHVU & 15212 & 21767 & 21979 & 30 & 33731 & 345 & 38 & & 62029 & & & & 134176 \\
\hline & AHVW & & & & & 5498 & & 5280 & 5869 & 6761 & 10444 & 16687 & 20689 & 16757 \\
\hline Othe & RKPL & 36352 & 306 & 32978 & 47136 & 82273 & $90 \varepsilon$ & & 824901 & 1072291 & 152170 & .. & .. & \\
\hline Total liabilities & GQFX & 4412 & 5347 & 8118 & 7436 & 8719 & 11041 & 10819 & 10005 & 26944 & 39626 & .. & .. & \\
\hline
\end{tabular}

1 These figures cover funded schemes only and therefore exclude the main

Source: Office for National Statistics: 01633812726 superannuation arrangements in the central government sector. 
Long-term insurance companies

Assets

Total current assets (gross)

Agents' and reinsurance balances (net)

Other debtors ${ }^{1}$

British government securities

UK local authority securities etc

UK company securities ${ }^{2}$

Overseas company securities

Overseas government securities

Loans and mortgages

UK land, property and ground rent

Overseas land, property and ground rent

Other investments

Total

Net value of direct investment in:

Non-insurance subsidiaries and associate companies in the United Kingdom

UK associate and subsidiary insurance

companies and insurance holding companies RYEU

$\begin{array}{cc}\text { companies and insurance holding companies } & \text { RYEU } \\ \text { Overseas subsidiaries and associates } & \text { RYEV }\end{array}$

Total assets

RKBI

$800792960922968481952882893588945808 \quad 1003640 \quad 1159307 \quad 12824271379232$

\begin{tabular}{|c|c|c|c|c|c|c|c|c|c|c|}
\hline RYEW & 46165 & 56360 & 62937 & 63855 & 58122 & 58518 & 63407 & 72754 & 77748 & 101780 \\
\hline AHNY & 1383 & 508 & 384 & 620 & 6373 & 4720 & 3755 & 3933 & 5100 & 984 \\
\hline RKPN & 18210 & 18613 & 21045 & 27285 & 34391 & 35414 & 30504 & 27591 & 52253 & 600 \\
\hline AHNJ & 127903 & 126223 & 116734 & 119513 & 131305 & 142920 & 157019 & 161906 & 161641 & 158694 \\
\hline AHNN & 1722 & 1456 & 1170 & 1407 & 1427 & 1547 & 2044 & 1840 & 1614 & \\
\hline RKPO & 438666 & 539834 & 557293 & 505691 & 443535 & 468910 & 487034 & 601681 & 643433 & 6647 \\
\hline RKPP & 82122 & 120665 & 107439 & 127259 & 110738 & 110193 & 130098 & 165452 & 194997 & 234 \\
\hline AHNS & 17515 & 18494 & 18004 & 21285 & 19762 & 20561 & 20161 & 16065 & 21078 & \\
\hline RKPQ & 11027 & 10914 & 9687 & 10048 & 10994 & 12107 & 12917 & 13502 & 15330 & 13 \\
\hline AHNX & 45903 & 50387 & 49705 & 53726 & 52658 & 57174 & 60502 & 61037 & 58918 & 66169 \\
\hline RGCP & 252 & 206 & 1975 & 498 & 158 & 184 & 94 & 27 & 61 & \\
\hline RKPR & 5654 & 8334 & 8385 & 7420 & 9513 & 17985 & 26480 & 18146 & 27783 & 3140 \\
\hline
\end{tabular}

RFXN

796522951994954760938609878979930233

\begin{tabular}{rrrrrrrrrrr}
3035 & 3045 & 6133 & 4486 & 4577 & 4191 & 3971 & 8390 & 13016 & 9186 \\
148 & 2245 & 3586 & 4206 & 4569 & 5054 & 3473 & 2528 & 6114 & 7578 \\
1087 & 3638 & 4002 & 5581 & 5463 & 6330 & 2181 & 4455 & 3341 & 3832 \\
\hline
\end{tabular}

\begin{tabular}{|c|c|c|c|c|c|c|c|c|c|c|}
\hline RGDF & 3252 & 6064 & 8272 & 8790 & 4958 & 4164 & 5358 & 5037 & 2862 & 3795 \\
\hline RGDE & 1040 & 3070 & 2823 & 5350 & 7406 & 10923 & 8385 & 9036 & 9542 & 6705 \\
\hline RGDD & 148 & 159 & 38 & 81 & 800 & 530 & 793 & 1151 & 1965 & 1926 \\
\hline RKDC & 669301 & 800184 & 838485 & 831051 & 794177 & 824766 & 873071 & 1037658 & 1125221 & 1205183 \\
\hline RKBM & 1712 & 2032 & 2249 & 2547 & 3234 & 3699 & 3579 & 3481 & 3513 & 3848 \\
\hline RYPI & 5443 & 6344 & 5381 & 3951 & 2803 & 4055 & 4881 & 8225 & 7908 & 7457 \\
\hline RYPJ & 67 & 314 & 67 & 45 & -20 & 2 & -13 & -2 & 199 & 5 \\
\hline RYPK & 359 & 201 & 183 & 87 & 32 & 27 & 93 & 22 & 13 & 27 \\
\hline RYPL & 12509 & 17042 & 19031 & 18468 & 23261 & 15870 & 16738 & 16907 & 33192 & 39527 \\
\hline RKBR & 96456 & 116951 & 79173 & 63337 & 36517 & 62546 & 65641 & 59132 & 71017 & 75066 \\
\hline RKTI & .. & 25 & .. & .. & .. & 1 & 267 & .. & .. & 192 \\
\hline RKBS & 6299 & 6139 & 10287 & 17044 & 18629 & 15698 & 20719 & 18717 & 27315 & 35855 \\
\hline RKBT & 4206 & 2396 & 2492 & 2130 & 1791 & 3527 & 4129 & -57 & -320 & -35 \\
\hline RKBI & 800792 & 960922 & 968481 & 952882 & 893588 & 945808 & 1003640 & 1159307 & 1282427 & 1379232 \\
\hline
\end{tabular}


Banking, insurance

1999

2000

2001

2002

2003

2004

2005

$2006 \quad 2007$

Other than long-term insurance companies

Assets

Total current assets (gross)

Agents' and reinsurance balances (net)

Other debtors ${ }^{1}$

British government securities

UK local authority securities etc

UK company securities ${ }^{2}$

Overseas company securities

Overseas government securities

Loans and mortgages

UK land, property and ground rent

Overseas land, property and ground rent

Other investments

Total

\begin{tabular}{|c|c|c|c|c|c|c|c|c|c|c|}
\hline RYME & 8524 & 10468 & 8772 & 12264 & 17671 & 20036 & 29258 & 26561 & 24942 & 25810 \\
\hline AHMX & 10528 & 12177 & 8362 & 7941 & 9492 & 9890 & 9858 & 7996 & 10782 & 9932 \\
\hline RKPS & 6277 & 7059 & 7179 & 9056 & 14437 & 13255 & 12618 & 13310 & 16881 & 20351 \\
\hline AHMJ & 16409 & 15938 & 14561 & 15064 & 18390 & 19645 & 19662 & 19818 & 19296 & 16026 \\
\hline AHMN & 14 & 10 & 8 & 6 & 10 & 10 & 49 & 44 & & \\
\hline RKPT & 18440 & 18800 & 18585 & 17101 & 15362 & 15153 & 20561 & 21879 & 22983 & 23812 \\
\hline RKPU & 8676 & 6284 & 8190 & 6402 & 7394 & 7124 & 11520 & 12645 & 18636 & 14773 \\
\hline AHMS & 10459 & 7980 & 6849 & 7134 & 7156 & 5720 & 6662 & 7341 & 8035 & 4869 \\
\hline RKPV & 1335 & 1070 & 1429 & 1348 & 1063 & 1400 & 2412 & 3040 & 3319 & 3684 \\
\hline AHMW & 1146 & 1085 & 1069 & 860 & 805 & 859 & 893 & 1470 & 1569 & 187 \\
\hline RYNK & 107 & 83 & 45 & 4 & 1 & 4 & 5 & 13 & 137 & 11 \\
\hline RKPW & 2366 & 2638 & 2294 & 1608 & 2182 & 1408 & 1858 & 2083 & 2010 & 188 \\
\hline RKAL & 84281 & 84027 & 77343 & 78789 & 93965 & 94504 & 115356 & 116200 & 128590 & 12312 \\
\hline RYNR & 5553 & 7074 & 7038 & 10456 & 11706 & 13408 & 19028 & 20530 & 20111 & 2195 \\
\hline RYNS & 6424 & 5617 & 5400 & 8837 & 7190 & 2918 & 2280 & 6071 & 4745 & 693 \\
\hline RYNT & 14239 & 17775 & 15993 & 14260 & 9014 & 5718 & 5507 & 6446 & 9657 & 944 \\
\hline RKBY & 110497 & 114493 & 105774 & 112342 & 121875 & 116548 & 142171 & 149247 & 163103 & 16146 \\
\hline
\end{tabular}

Net value of direct investment in

Non-insurance subsidiaries and associate companies in the United Kingdom

UK associate and subsidiary insurance

companies and insurance holding companies

Overseas subsidiaries and associates

110497

Total assets

\section{Liabilities}

Borrowing:

Borrowing from UK banks

Other UK borrowing

Borrowing from overseas

General business technical reserves

Long-term business:

Funds

Claims admitted but not paid

Provision for taxation net of amounts receivable: UK authorities

Overseas authorities

Provision for recommended dividends

Other creditors and liabilities

Excess of assets over above liabilities:

Excess of value of assets over liabilities in respect of long-term funds

Minority interests in UK subsidiary companies

Shareholders' capital and reserves in respect of general business

Other reserves including profit and loss account balances

\begin{tabular}{|c|c|c|c|c|c|c|c|c|c|c|}
\hline RYMB & 1825 & 1392 & 783 & 481 & 1384 & 2046 & 4519 & 893 & 3148 & 675 \\
\hline RYMC & 1551 & 3186 & 4239 & 10621 & 10472 & 9342 & 10261 & 11080 & 10445 & 10885 \\
\hline RYMD & 1600 & 3045 & 1867 & 1964 & 2916 & 2918 & 2476 & 2817 & 5459 & 7037 \\
\hline RKCT & 60775 & 59455 & 60236 & 60995 & 62776 & 63463 & 67241 & 71710 & 77221 & 71146 \\
\hline RKTF & - & - & - & - & - & - & - & - & - & \\
\hline RKTK & - & - & - & - & - & - & - & - & - & \\
\hline YPO & 1197 & 939 & 874 & 594 & 941 & 834 & 1094 & 1796 & 2376 & 2259 \\
\hline RYPP & 11 & 11 & 11 & 7 & 5 & 84 & 24 & 5 & 10 & \\
\hline RYPQ & 1318 & 1817 & 2682 & 1957 & 958 & 1082 & 1311 & 5 & 270 & 222 \\
\hline RYPR & 3793 & 4981 & 6293 & 6410 & 8025 & 9567 & 10817 & 10718 & 16226 & 22069 \\
\hline RKCG & - & - & - & - & - & - & - & - & - & \\
\hline $\mathrm{RKCH}$ & 68 & 29 & 33 & 276 & 4 & 6 & 6 & .. & .. & 599 \\
\hline RKCI & 34397 & 35372 & 24699 & 26190 & 31982 & 25153 & 39695 & 43264 & 42186 & 38145 \\
\hline RKCJ & 4215 & 4265 & 4056 & 2847 & 2411 & 2053 & 4727 & 6959 & 5762 & 8421 \\
\hline KBY & 110497 & 114493 & 105774 & 112342 & 121875 & 116548 & 142171 & 149247 & 163103 & 161463 \\
\hline
\end{tabular}

Total liabilities

Source: Office for National Statistics: 0163381272

1 Including outstanding interest, dividends and rents (net).

2 Including authorised unit trust units. 


\begin{tabular}{|c|c|c|c|c|c|c|c|c|c|c|c|c|}
\hline & & 1998 & 1999 & 2000 & 2001 & 2002 & 2003 & 2004 & 2005 & 2006 & 2007 & 2008 \\
\hline \multicolumn{13}{|l|}{ England and Wales } \\
\hline \multirow{2}{*}{$\begin{array}{l}\text { Bankruptcies }{ }^{1} \\
\text { Individual voluntary } \\
\text { arrangements }{ }^{2,3}\end{array}$} & AIHW & 19647 & 21611 & 21550 & 23477 & 24292 & 28021 & 35898 & 47291 & 62956 & 64480 & 67428 \\
\hline & AIHI & 4902 & 7195 & 7978 & 6298 & 6295 & 7583 & 10752 & 20293 & 44332 & 42165 & 39116 \\
\hline Total & AIHK & 24549 & 28806 & 29528 & 29775 & 30587 & 35604 & 46650 & 67584 & 107288 & 106645 & 106544 \\
\hline \multicolumn{13}{|l|}{ Scotland } \\
\hline $\begin{array}{l}\text { Sequestrations }{ }^{4} \\
\text { Protected Trust Deeds }\end{array}$ & $\begin{array}{l}\text { KRHA } \\
\text { GJ2I }\end{array}$ & $\begin{array}{l}3016 \\
1449\end{array}$ & $\begin{array}{l}3195 \\
2144\end{array}$ & $\begin{array}{l}2965 \\
2801\end{array}$ & $\begin{array}{l}3048 \\
3779\end{array}$ & $\begin{array}{l}3215 \\
5174\end{array}$ & $\begin{array}{l}3328 \\
5452\end{array}$ & $\begin{array}{l}3297 \\
6024\end{array}$ & $\begin{array}{l}4965 \\
6881\end{array}$ & $\begin{array}{l}5430 \\
8208\end{array}$ & $\begin{array}{l}6219 \\
7595\end{array}$ & $\begin{array}{r}12322 \\
7542\end{array}$ \\
\hline Total & GJ2J & 4465 & 5339 & 5766 & 6827 & 8389 & 8780 & 9321 & 11846 & 13638 & 13814 & 19864 \\
\hline \multirow{2}{*}{$\begin{array}{l}\text { Bankruptcies }{ }^{5} \\
\text { Individual voluntary } \\
\text { arrangements }\end{array}$} & KRHB & 394 & 401 & 349 & 292 & 334 & 517 & 666 & 821 & 1035 & 898 & 1079 \\
\hline & KJRK & 123 & 172 & 267 & 176 & 207 & 318 & 449 & 633 & 774 & 440 & 559 \\
\hline Total & KRHD & 517 & 573 & 616 & 468 & 541 & 835 & 1115 & 1454 & 1809 & 1338 & 1638 \\
\hline
\end{tabular}

1 Comprises receiving and administration orders under the Bankruptcy Act 5 Comprises bankruptcy adjudication orders, arrangement protection orders and 1914 and bankruptcy orders under the Insolvency Act 1986. Orders later orders for the administration of estates of deceased insolvents. Orders later set consolidated or rescinded are included in these figures. aside or dismissed are included in these figures.

2 Introduced under the Insolvency Act 1986

3 For statistical purposes deeds of arrangement are now included with individual voluntary arrangements.

Introduced under the Insolvency Northern Ireland order 1989.

Sequestrations awarded but not brought into operation are included in

these figures.

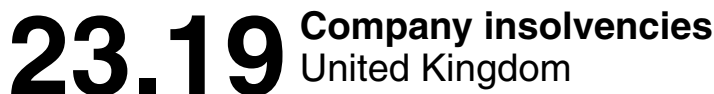

Numbers

\begin{tabular}{|c|c|c|c|c|c|c|c|c|c|c|c|c|}
\hline & & 1998 & 1999 & 2000 & 2001 & 2002 & 2003 & 2004 & 2005 & 2006 & 2007 & 2008 \\
\hline \multicolumn{13}{|l|}{ England and Wales } \\
\hline liquidations & AIHS & 7987 & 9071 & 9392 & 10297 & 10075 & 8950 & 7608 & 7660 & 7719 & 7342 & 10041 \\
\hline Total & AIHQ & 13203 & 14280 & 14317 & 14972 & 16306 & 14184 & 12192 & 12893 & 13137 & 12507 & 15535 \\
\hline Scotland & & & & & & & & & & & & \\
\hline $\begin{array}{l}\text { Compulsory liquidations } \\
\text { Creditors' voluntary }\end{array}$ & KRGA & 338 & 364 & 344 & 378 & 556 & 436 & 431 & 420 & 416 & 439 & 437 \\
\hline liquidations & KRGB & 228 & 208 & 239 & 224 & 232 & 195 & 190 & 149 & 133 & 100 & 87 \\
\hline Total & KRGC & 566 & 572 & 583 & 602 & 788 & 631 & 621 & 569 & 549 & 539 & 524 \\
\hline $\begin{array}{l}\text { Compulsory liquidations } \\
\text { Creditors' voluntary }\end{array}$ & KRGD & .. & .. & .. & .. & 49 & 95 & 76 & 85 & 78 & 122 & 158 \\
\hline liquidations & KRGE & .. & .. & .. & .. & 53 & 47 & 45 & 53 & 50 & 42 & 51 \\
\hline Total & KRGF & .. & .. & .. & .. & 102 & 142 & 121 & 138 & 128 & 164 & 209 \\
\hline
\end{tabular}

1 Prior to 2002, the quality of the statistics on company liquidations in

Northern Ireland are not robust enough and have been removed from this

Source: Insolvency Service: $02076376504 / 6443$ table. 


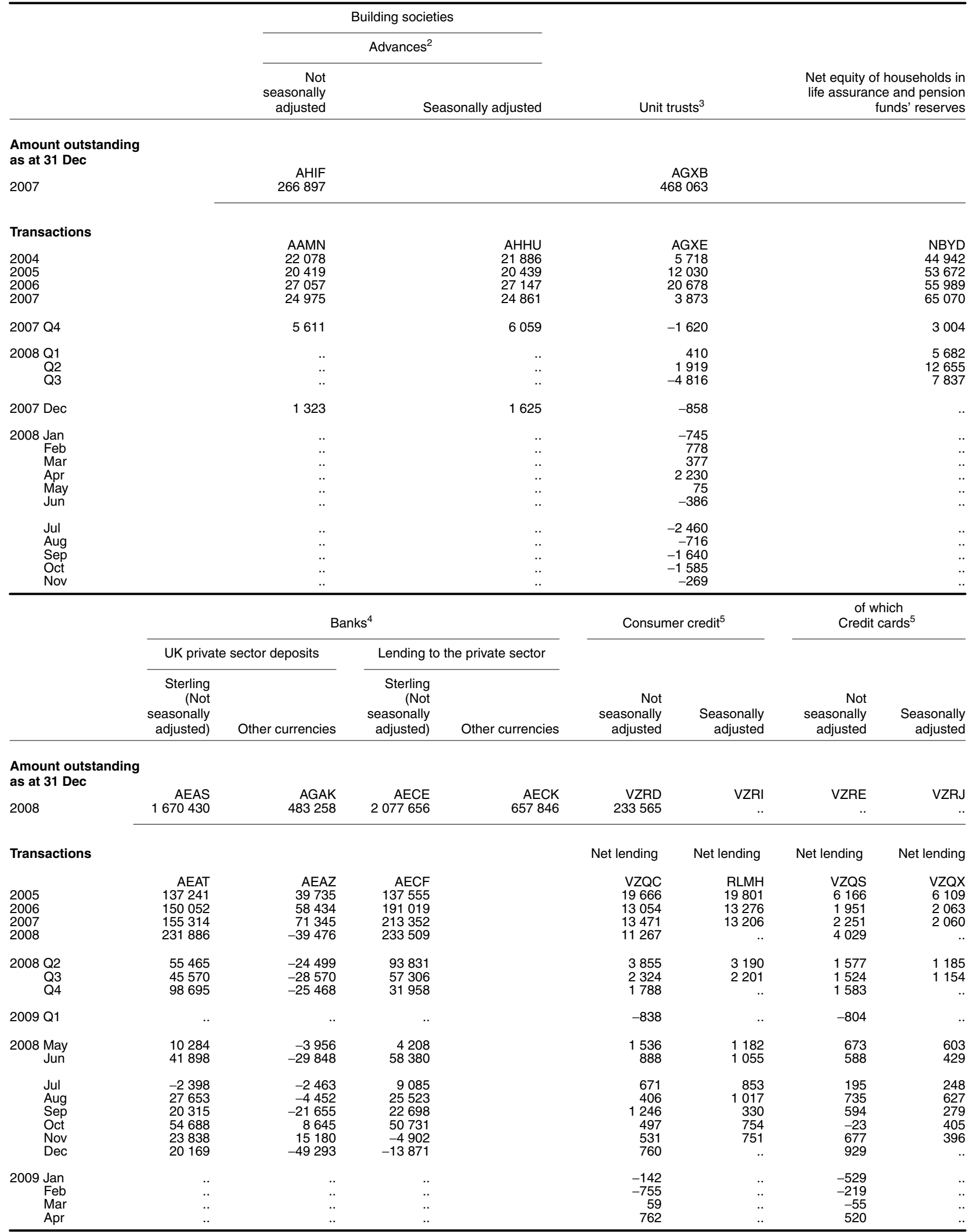

1 For further details see Financial Statistics, Tables 1.2F, 3.2B, 4.2A, 4.3A, 5 Data have been revised back to February 2003 due to the inclusion of some 4.3B, 5.2D, 6.2A, 10.5D.

2 Total administered by the Department for National Savings.

3 Including open ended investment companies (OEICs). additional other specialist lenders and the removal of some non-resident based securitisation vehicles.

4 Monthly figures relate to calendar months.

Sources: Office for National Statistics; Department for National Savings; Building Societies Commission; Association of Unit Trusts and Investment Funds; Bank of England; 


\subsection{Selected interest rates, exchange rates and security prices}

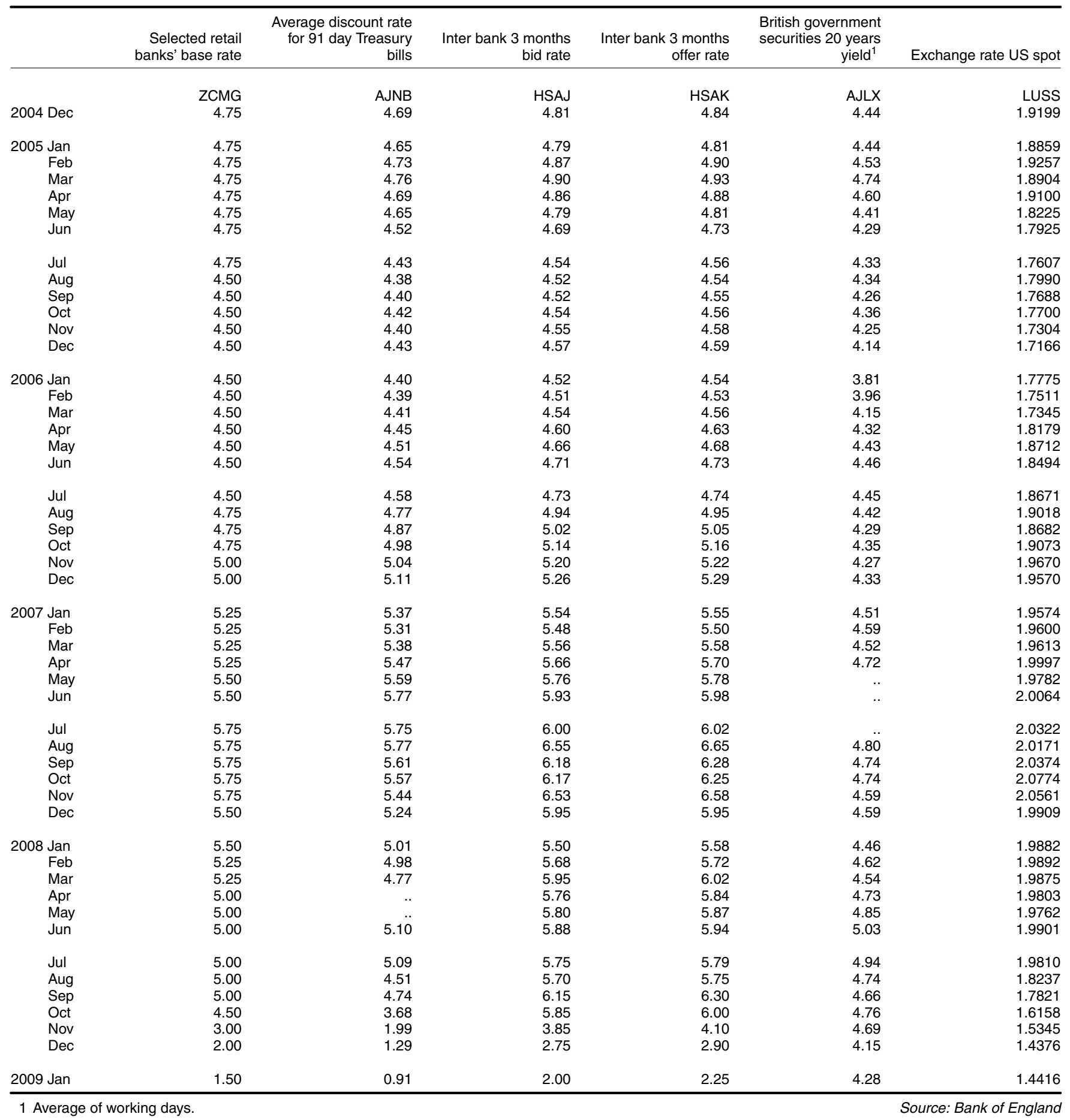




\begin{tabular}{|c|c|c|c|c|c|c|}
\hline & \multirow[b]{3}{*}{$\begin{array}{r}\text { Number of companies } \\
\text { acquired }\end{array}$} & \multicolumn{5}{|c|}{ Expenditure } \\
\hline & & \multirow[b]{2}{*}{ Total $^{1}$} & \multicolumn{2}{|c|}{ Cash } & \multirow[b]{2}{*}{$\begin{array}{l}\text { Issues of ordinary } \\
\text { shares }^{2}\end{array}$} & \multirow{2}{*}{$\begin{array}{r}\text { Issues of } \\
\text { fixed interest } \\
\text { securities }^{2}\end{array}$} \\
\hline & & & $\begin{array}{r}\text { Independent } \\
\text { companies }\end{array}$ & Subsidiaries & & \\
\hline & AlHA & DUCM & DWVW & DWVX & AlHD & AlHE \\
\hline 1998 & 635 & 29525 & 10471 & 5298 & 13160 & 595 \\
\hline 1999 & 493 & 26163 & 12605 & 3615 & 9592 & 351 \\
\hline 2000 & 587 & 106916 & 33906 & 6168 & 65570 & 1272 \\
\hline 2001 & 492 & 28994 & 8489 & 6704 & 12356 & 1445 \\
\hline 2002 & 430 & 25236 & 9574 & 7991 & 6780 & 891 \\
\hline 2003 & 558 & 18679 & 8956 & 7183 & 1667 & 873 \\
\hline 2004 & 741 & 31408 & 12080 & 7822 & 10338 & 1168 \\
\hline 2005 & 769 & 25134 & 13425 & 8510 & 2768 & 431 \\
\hline 2006 & 779 & 28511 & & 8131 & & 335 \\
\hline 2007 & 869 & 26778 & 13671 & 6507 & 4909 & 1691 \\
\hline 1998 Q4 & 162 & 6586 & 2090 & 1181 & 3255 & 59 \\
\hline 1999 Q1 & 117 & 8735 & 2299 & 625 & 5735 & 76 \\
\hline Q2 & 127 & 7212 & 4893 & 728 & 1509 & 82 \\
\hline Q3 & 145 & 6479 & 2618 & 1682 & 2098 & 81 \\
\hline Q4 & 104 & 3737 & 2795 & 580 & 250 & 112 \\
\hline 2000 Q1 & 139 & 33739 & 17483 & 1136 & 14960 & 160 \\
\hline Q2 & 133 & 21469 & 4224 & 1881 & 15045 & 319 \\
\hline Q3 & 163 & 16852 & 6934 & 2237 & 7367 & 314 \\
\hline Q4 & 152 & 34856 & 5265 & 914 & 28198 & 479 \\
\hline 2001 Q1 & 131 & 6181 & 2606 & 2255 & 982 & 338 \\
\hline Q2 & 108 & 4890 & 1679 & 2214 & 555 & 442 \\
\hline Q3 & 129 & 16079 & 3457 & 1526 & 10649 & 447 \\
\hline Q4 & 124 & 1844 & 747 & 709 & 170 & 218 \\
\hline 2002 Q1 & 83 & 3853 & 2201 & 1298 & 104 & 250 \\
\hline Q2 & 120 & 4228 & 801 & 3179 & 78 & 170 \\
\hline Q3 & 88 & 6333 & 4695 & 1426 & 184 & 28 \\
\hline Q4 & 139 & 10822 & 1877 & 2088 & 6414 & 443 \\
\hline Q3 & 153 & 4700 & 2495 & 1919 & 153 & 133 \\
\hline Q4 & 176 & 6369 & 4021 & 1659 & 647 & 42 \\
\hline 2004 Q1 & 151 & 12639 & 2819 & 655 & 8807 & 358 \\
\hline Q2 & 169 & 5359 & 2555 & 1682 & 822 & 300 \\
\hline Q3 & 211 & 8109 & 3469 & 4026 & 240 & 374 \\
\hline Q4 & 210 & 5301 & 3237 & 1459 & 469 & 136 \\
\hline 2005 Q1 & 166 & 3516 & 1334 & 1918 & 166 & 98 \\
\hline Q2 & 215 & 8983 & 4869 & 2715 & 1285 & 114 \\
\hline Q3 & 211 & 7287 & 4106 & 1878 & 1207 & 96 \\
\hline Q4 & 177 & 5348 & 3116 & 1999 & 110 & 123 \\
\hline 2006 Q1 & 207 & 6969 & 4069 & 2427 & 431 & 42 \\
\hline Q2 & 208 & 4222 & 3298 & 527 & 384 & 13 \\
\hline Q3 & 163 & 11376 & & 4580 & & 216 \\
\hline Q4 & 201 & 5944 & 4690 & 597 & 593 & 64 \\
\hline 2007 Q1 & 191 & 5649 & 2824 & 276 & 2407 & 142 \\
\hline Q2 & 212 & 10122 & 3605 & 4361 & 1874 & 282 \\
\hline Q3 & 258 & 7846 & 5545 & 833 & 358 & 1110 \\
\hline Q4 & 208 & 3161 & 1697 & 1037 & 270 & 157 \\
\hline 2008 Q1 & 167 & 4512 & 2566 & 906 & 772 & 268 \\
\hline Q2 & 174 & 9270 & 8697 & 383 & 150 & 40 \\
\hline Q3 & 79 & 3902 & 3260 & 304 & 285 & 53 \\
\hline
\end{tabular}

Missing data for any series have been suppressed to avoid the disclosure of information relating to individual enterprises.

2 Issued to the vendor as payment. 